\title{
Fractional optimal control in transmission dynamics of West Nile virus model with state and control time delay: a numerical approach
}

\author{
N.H. Sweilam ${ }^{1 *}$ (D, O.M. Saad ${ }^{2}$ and D.G. Mohamed ${ }^{2}$
}

\section{"Correspondence:}

nsweilam@sci.cu.edu.eg

'Department of Mathematics,

Faculty of Science, Cairo University, Giza, Egypt

Full list of author information is available at the end of the article

\begin{abstract}
In this paper, an optimal control for a novel fractional West Nile virus model with time delay is presented. The proposed model is governed by a system of fractional delay differential equations, where the fractional derivative is defined in the Grünwald-Letnikov sense. Stability analysis of fixed points is studied. Corresponding fractional optimal control problem, with time delays in both state and control variables, is formulated and studied. Two simple numerical methods are used to study the nonlinear fractional delay optimal control problem. The methods are standard finite difference method and nonstandard finite difference method. Comparative studies are implemented, it is found that the nonstandard finite difference method is better than the standard finite difference method.
\end{abstract}

MSC: 49J15; 37N25; 26A33; 93C15; 65L12

Keywords: West Nile virus mathematical model; Delay differential equations; Nonstandard finite difference method; Grünwald-Letnikov's definition; Fractional optimal control problem

\section{Introduction}

Time delays play an important role in the modeling of real-life phenomena in different fields of applications, for example, engineering, chemical, and biological systems that are modeled by differential equations. Many methodologies have been proposed to deal with the control and optimal control problems of systems with time delay [1-9]. In [10], an introduction to time-delay control problems is given. In [11], Kharatishvili established a maximum principle for optimal control problems with a constant state delay. Similar results for control problems with pure control delays are presented in [12]. In [13], Halanay proved a maximum principle for optimal control problems with multiple constant delays in state and control variables. Soliman and Ray obtained the similar results in [14]. A simple method for obtaining necessary conditions for control problems with a constant delay in the state variable is introduced in [15]. A maximum principle for control systems with a time-dependent delay in the state variable is derived in [16]. The theory of necessary conditions for optimization problems in functional spaces is applied on a unified approach to control problems with delays in the state variable in [17]. Optimal control for a time-delay

(c) The Author(s) 2019. This article is distributed under the terms of the Creative Commons Attribution 4.0 International License (http://creativecommons.org/licenses/by/4.0/), which permits unrestricted use, distribution, and reproduction in any medium, provided you give appropriate credit to the original author(s) and the source, provide a link to the Creative Commons license, and indicate if changes were made. 
multi-strain tuberculosis fractional model is introduced in [18]. Recently, many interesting studies were presented in nonlinear and time-delay optimal control problems, see [19-21].

Fractional calculus has been the focus of many studies due to its appearance in various sciences [22-30]. The paths of the control variables that maximize or minimize a function of the state and control variables are described by fractional optimal control problems (FOCP). The theory of FOCPs has been under development. The approximation and numerical techniques are used to solve the FOCPs [25, 31]. Since most of the fractional-order differential equations do not have exact analytic solutions, the approximation and numerical techniques must be used [26]. Delayed fractional differential equations (DEDEs) are also used to describe dynamical systems [32], for more details, see [33-35]. Recently, many papers have been devoted to DEDEs (see [36-38], and the references cited therein). Nonstandard finite difference method (NSFDM) was firstly developed in 1980s by Mickens and has been applied in many areas of science including biological and epidemic models [39-41]. It is important that the proposed numerical method can preserve the positivity of the solution [42].

In this paper, fractional time-delay optimal control in transmission dynamics of WNV model is presented as an extension of the WNV model given in [26]. Time delays in both state and control variables are formulated and studied. The aim of this paper is to study the fractional time-delay optimal control of this proposed model. We introduced NSFDM and standard finite difference method (SFDM) to study the behavior of this optimal control problem.

West Nile virus (WNV) is an arbovirus and single-standard RNA virus Flavivirus [43]. WNV is transmitted among mosquitoes, birds, humans, and other animals [44]. In 1937, the virus was first isolated in the West Nile district of Uganda [45]. It has spread in Africa, Europe, the Middle East, west and central Asia, Oceania (subtype Kunjin), and North America [46-50]. Owing to the absence of an effective diagnostic test therapeutic treatment against WNV, public health officials have focussed on the use of preventive measures in attempt to halt the spread of WNV in humans [43]. In 2001 [51], the first WNV model was presented by Thomas and Urena. In [52], a single season model with a system of differential equations for WNV transmission in the mosquito-bird population was presented by Wonham et al. They were using local stability results and simulations, showed that while mosquito control decreases WNV outbreak threshold, controlling birds increases it. In [53] Cruz-Pacheco et al. studied a mathematical model for the transmission of WNV infection between mosquito and avain populations, and by using experimental and field data as well as numerical simulations, they found the phenomenon of damped oscillations of the infected bird population. Also, the spatial spread of the virus was established by Lewis et al. in [54]. A comparative study of the discrete-time model in [51] and the continuoustime model in [55] was introduced by Lewis et al. in [56]. Kbenesh et al. determined the cost-effective strategies for combating the spread of WNV in a given population in [57]. Fractional optimal control of WNV model was introduced by using two numerical methods by Sweilam et al. in [26].

This paper is organized as follows. In Sect. 2, preliminaries and notations are presented. In Sect. 3, a mathematical model is formulated. Reproduction number $R_{0}$ and stability of the disease-free equilibrium and the endemic equilibrium are studied in Sect. 4 and Sect. 5, respectively. The optimal control problem is formulated in Sect. 6. In Sect. 7, NSFD 
method for fractional time-delay WNV model is presented. A numerical experiment is discussed in Sect. 8. Finally, in Sect. 9, conclusions are presented.

\section{Preliminaries and notations}

Definition 2.1 ([58]) The left Caputo fractional derivative (LCFD) is defined as follows:

$$
{ }_{a}^{c} D_{t}^{\alpha} \mathcal{V}(t)=\frac{1}{\Gamma(n-\alpha)} \int_{a}^{t} \frac{\mathcal{V}^{(n)}(\tau)}{(t-\tau)^{1-n+\alpha}} d \tau,
$$

the right Caputo fractional derivative (RCFD) is defined as follows:

$$
{ }_{t}^{c} D_{b}^{\alpha} \mathcal{V}(t)=\frac{(-1)^{n}}{\Gamma(n-\alpha)} \int_{t}^{b} \frac{\mathcal{V}^{(n)}(\tau)}{(t-\tau)^{1-n+\alpha}} d \tau
$$

where $\Gamma$ is the Euler gamma function.

Definition 2.2 ([58]) The Grünwald-Letnikov approximation of the fractional derivative of order $\alpha$ of the function $\mathcal{V}(t)$ is defined as follows:

$$
D^{\alpha} \mathcal{V}(t)=\lim _{h \rightarrow 0}(h)^{-\alpha} \sum_{k=0}^{\left[\frac{t}{h}\right]} \omega_{k}^{\alpha} \mathcal{V}(t-k h),
$$

where $[t]$ is the integer part of $t, h$ is the step size, and the Grünwald-Letnikov coefficients are given by

$$
\omega_{k}^{\alpha}=\left(1-\frac{\alpha+1}{k}\right) \omega_{k-1}^{\alpha}, \quad \text { and } \quad \omega_{0}^{\alpha}=(h)^{-\alpha}, \quad k>1 .
$$

Definition 2.3 ([59]) The numerical scheme is called NSFD discretization if at least one of the following conditions is satisfied:

(i) Nonlocal approximation is used [39, 59, 60].

(ii) The discretization of derivative is not traditional and a nonnegative function $\varphi(\Delta t)=\Delta t+O\left((\Delta t)^{2}\right)$, called a denominator function, is used [41].

The discretization is based on the approximations of temporal derivatives by a generalized forward scheme. If we have $\varphi(\Delta t)=\Delta t$, then the scheme is defined as finite difference or SFDM. Hence, if $\mathcal{M}(t) \in C^{1}(\mathbb{R})$, the first derivative can be defined as follows:

$$
\frac{d \mathcal{M}(t)}{d t}=\frac{\mathcal{M}(t+\Delta t)-\mathcal{M}(t)}{\varphi(\Delta t)} .
$$

\section{Mathematical model}

In this section, a new formulation for the transmission dynamics of WNV model given in [57] is presented. In the following, the transmission dynamics of WNV model is modified to be nonlinear time-delayed fractional differential equations. The WNV introduced in [57] consists of nine nonlinear ODEs which is described by the total mosquito population at time $t$, denoted by $N_{M}(t)$, split into the populations of susceptible $M_{s}(t)$ and infected $M_{i}(t)$ mosquitoes. Similarly, the total bird population at time $t$, denoted by $N_{B}(t)$, is subdivided into susceptible $B_{s}(t)$ and infected $B_{i}(t)$ birds. Finally, the total human population 
Table 1 All variables in system (6) and their definitions

\begin{tabular}{ll}
\hline Variable & Definition \\
\hline$M_{S}(t)$ & The population of susceptible mosquitoes \\
$M_{i}(t)$ & The population of infected mosquitoes \\
$N_{M}(t)$ & The total population of mosquitoes $N_{M}(t)=M_{S}(t)+M_{i}(t)$ \\
$B_{S}(t)$ & The population of susceptible birds \\
$B_{i}(t)$ & The population of infected birds \\
$N_{B}(t)$ & The total population of birds $N_{B}(t)=B_{S}(t)+B_{i}(t)$ \\
$S(t)$ & The population of susceptible humans \\
$E(t)$ & The population of exposed humans \\
$I(t)$ & The population of infected humans \\
$H(t)$ & The population of hospitalized humans \\
$R(t)$ & The population of recovered humans \\
$N_{H}(t)$ & The total population of humans $N_{H}(t)=S(t)+E(t)+I(t)+H(t)+R(t)$ \\
\hline
\end{tabular}

at time $t$, denoted by $N_{H}(t)$, is split into susceptible $S(t)$, exposed $E(t)$, infectious $I(t)$, hospitalized $H(t)$, and recovered $R(t)$ humans. Thus, $N_{M}(t)=M_{s}(t)+M_{i}(t), N_{B}(t)=B_{s}(t)+B_{i}(t)$ and $N_{H}(t)=S(t)+E(t)+I(t)+H(t)+R(t)$ as explained in Table 1. The population of susceptible humans is increased via recruitment of humans (by birth or immigration) into the community at a constant rate $\lambda_{H}$. It is decreased infection (acquired via contact with infected mosquitoes) and by natural death (at a rate $\lambda_{H}$ ), where $b_{2}$ is the per capita rate of biting of humans by mosquitoes and $\beta_{3}$ is the probability of WNV transmission from mosquitoes to humans. Here, it is assumed that new human infections are acquired at a rate that depends on the average number of mosquito bites per unit time and on the transmission probability normalized by total human population [43]. Let us introduce a discrete time delay in the state variables $M_{i}(t), S(t)$, and $H(t)$, denoted by $\mathbf{d}_{\mathbf{d}}, \mathbf{d}_{\mathbf{d}}$, and $\mathbf{d}_{\mathbf{H}}$, respectively (where $\mathbf{d}_{\mathbf{d}}$ represents the time required for the WNV transmission from mosquitos to humans and $\mathbf{d}_{\mathbf{H}}$ represents the time required for diagnosis and commencement of treatment of the infection of WNV model). The time-delay system of WNV model of fractional-order derivatives is

$$
\begin{aligned}
& { }_{a}^{c} D_{t}^{\alpha} M_{s}(t)=\lambda_{M}^{\alpha}-\frac{b_{1}^{\alpha} \beta_{1}^{\alpha} M_{s}(t) B_{i}(t)}{N_{B}(t)}-\mu_{M}^{\alpha} M_{s}(t), \\
& { }_{a}^{c} D_{t}^{\alpha} M_{i}(t)=\frac{b_{1}^{\alpha} \beta_{1}^{\alpha} M_{s}(t) B_{i}(t)}{N_{B}(t)}-\mu_{M}^{\alpha} M_{i}(t), \\
& { }_{a}^{c} D_{t}^{\alpha} B_{s}(t)=\lambda_{B}^{\alpha}-\frac{b_{1}^{\alpha} \beta_{2}^{\alpha} M_{i}(t) B_{s}(t)}{N_{B}(t)}-\left(\Psi_{B}^{\alpha}+\mu_{B}^{\alpha}\right) B_{s}(t), \\
& { }_{a}^{c} D_{t}^{\alpha} B_{i}(t)=\frac{b_{1}^{\alpha} \beta_{2}^{\alpha} M_{i}(t) B_{s}(t)}{N_{B}(t)}-\left(d_{B}^{\alpha}+\Psi_{B}^{\alpha}+\mu_{B}^{\alpha}\right) B_{i}(t), \\
& { }_{a}^{c} D_{t}^{\alpha} S(t)=\lambda_{H}^{\alpha}-\frac{b_{2}^{\alpha} \beta_{3}^{\alpha} M_{i}\left(t-\mathbf{d}_{\mathbf{d}}\right) S\left(t-\mathbf{d}_{\mathbf{d}}\right)}{N_{H}(t)}-\mu_{H}^{\alpha} S(t), \\
& { }_{a}^{c} D_{t}^{\alpha} E(t)=\frac{b_{2}^{\alpha} \beta_{3}^{\alpha} M_{i}\left(t-\mathbf{d}_{\mathbf{d}}\right) S\left(t-\mathbf{d}_{\mathbf{d}}\right)}{N_{H}(t)}-\left(\alpha_{w}^{\alpha}+\mu_{H}^{\alpha}\right) E(t), \\
& { }_{a}^{c} D_{t}^{\alpha} I(t)=\alpha_{w}^{\alpha} E(t)-\left(\gamma^{\alpha}+d_{I}^{\alpha}+r^{\alpha}+\mu_{H}^{\alpha}\right) I(t), \\
& { }_{a}^{c} D_{t}^{\alpha} H(t)=\gamma^{\alpha} I(t)-\left(d_{H}^{\alpha}+\mu_{H}^{\alpha}\right) H(t)-\tau^{\alpha} H\left(t-\mathbf{d}_{\mathbf{H}}\right), \\
& { }_{a}^{c} D_{t}^{\alpha} R(t)=\tau^{\alpha} H\left(t-\mathbf{d}_{\mathbf{H}}\right)+r^{\alpha} I(t)-\mu_{H}^{\alpha} R(t),
\end{aligned}
$$


Table 2 All parameters in system (6) and their interpretation

\begin{tabular}{ll}
\hline Parameter & Interpretation \\
\hline$\lambda_{M}^{\alpha}$ & The recruitment rate of mosquitoes (assumed susceptible) \\
$\mu_{M}^{\alpha}$ & The natural death rate of mosquitoes \\
$\lambda_{B}^{\alpha}$ & The recruitment rate of birds (assumed susceptible) \\
$\mu_{B}^{\alpha}$ & The natural death rate of birds \\
$\Psi_{B}^{\alpha}$ & The migration rate of birds \\
$d_{B}^{\alpha}$ & The WNV-induced death rate of birds \\
$\lambda_{H}^{\alpha}$ & The recruitment rate of humans (assumed susceptible) \\
$\mu_{H}^{\alpha}$ & The natural death rate of humans \\
$d_{1}^{\alpha}$ & The WNV-induced death rate of humans \\
$d_{H}^{\alpha}$ & The death rate of hospitalized humans \\
$\beta_{1}^{\alpha}$ & The probability of WNV transmission from an infected bird to a susceptible mosquito \\
$\beta_{2}^{\alpha}$ & The probability of WNV transmission from an infected mosquito to a susceptible bird \\
$\beta_{3}^{\alpha}$ & The probability of WNV transmission from mosquitoes to humans \\
$b_{1}^{\alpha}$ & The per capita bitting rate of mosquitoes on the primary host (birds) and $b_{1}^{\alpha}=\frac{b^{\alpha} N_{B}}{N_{B}+N_{H}}$ \\
$b_{2}^{\alpha}$ & The per capita bitting rate of mosquitoes on the humans and $b_{2}^{\alpha}=\frac{b^{\alpha} N_{H}}{N_{B}+N_{H}}$ \\
$b^{\alpha}$ & The average bitting rate of mosquitoes \\
$\alpha_{\omega}^{\alpha}$ & The rate of development of clinical symptoms of WNV \\
$r^{\alpha}$ & The natural recovery rate \\
$\tau^{\alpha}$ & The treatment-induced recovery rate \\
$\gamma^{\alpha}$ & The hospitalized rate of infectious humans \\
\hline
\end{tabular}

Table 3 Parameter values used in system (6)

\begin{tabular}{llll}
\hline Parameter & Value & Parameter & Value \\
\hline$\mu_{H}^{\alpha}$ & $\left(\left(3.91 \times 10^{-5}\right)^{\alpha}-0.005^{\alpha}\right)$ & $d_{H}^{\alpha}$ & $\left(\left(5 \times 10^{-5}\right)^{\alpha}-0.015^{\alpha}\right)$ \\
$\mu_{B}^{\alpha}$ & $\left(0.0001^{\alpha}-0.0003^{\alpha}\right)$ & $d_{B}^{\alpha}$ & $\left(0.06^{\alpha}-0.2^{\alpha}\right)$ \\
$\mu_{M}^{\alpha}$ & $\left(0.016^{\alpha}-0.07^{\alpha}\right)$ & $\lambda_{M}^{\alpha}$ & $\left(51.1 \times 10^{-3}\right)^{\alpha}$ \\
$\lambda_{B}^{\alpha}$ & $2.1^{\alpha}$ & $\lambda_{H}^{\alpha}$ & $\left(5 \times 10^{-2}\right)^{\alpha}$ \\
$d_{1}^{\alpha}$ & $\left(d_{H}+10^{-5}\right)^{\alpha}$ & $\Psi_{B}^{\alpha}$ & $\left(5.2 \times 10^{-2}\right)^{\alpha}$ \\
$\beta_{1}^{\alpha}$ & $0.4^{\alpha}$ & $\beta_{2}^{\alpha}$ & $0.1^{\alpha}$ \\
$\beta_{3}^{\alpha}$ & $\left(10^{-2}\right)^{\alpha}$ & $b^{\alpha}$ & $3^{\alpha}$ \\
$\alpha_{\omega}^{\alpha}$ & $0.1^{\alpha}$ & $r^{\alpha}$ & $\left(2 \times 10^{-4}\right)^{\alpha}$ \\
$\tau^{\alpha}$ & $0.05^{\alpha}$ & $\gamma^{\alpha}$ & $\left(9 \times 10^{-4}\right)^{\alpha}$ \\
\hline
\end{tabular}

with the following initial conditions:

$$
\begin{array}{lll}
M_{s}(\eta)=\zeta_{1}(\eta), & M_{i}(\eta)=\zeta_{2}(\eta), & B_{s}(\eta)=\zeta_{3}(\eta), \\
B_{i}(\eta)=\zeta_{4}(\eta), & S(\eta)=\zeta_{5}(\eta), & E(\eta)=\zeta_{6}(\eta), \\
I(\eta)=\zeta_{7}(\eta), & H(\eta)=\zeta_{8}(\eta), & R(\eta)=\zeta_{9}(\eta),
\end{array}
$$

where ${ }_{a}^{c} D_{t}^{\alpha}$ is the Caputo fractional-order derivative. Let us consider that $\mathbf{d}_{\mathbf{d}}=\mathbf{d}_{\mathbf{H}}=\mathbf{d}$. Since $\zeta \in[-\mathbf{d}, 0]$, where $\zeta=\left(\zeta_{1}, \zeta_{2}, \ldots, \zeta_{9}\right)^{T} \in \mathbb{C}$, and $\mathbb{C}$ is the Banach space $\mathbb{C}\left([0, \mathbf{d}], \mathbb{R}^{9}\right)$. The variables in system (6) are defined in Table 1 . The list of parameter values and their interpretation are introduced in Tables 2 and 3; for more details, see [57].

\section{Reproduction number $R_{0}$ and stability of the disease-free equilibrium}

In this section, we compute the reproduction number $R_{0}$. The basic reproduction number is the expected number of secondary cases produced, in a completely susceptible population, by a typical infective individual [32]. Method in [32] is applied to derive $R_{0}$. The 
order of infected variables is

$$
\varsigma=\left(M_{i}, B_{i}, E, I, H, R\right)^{\prime}
$$

The new infections into the infected classes $\zeta$ are defined by the vector $\mathbf{F}$, and the other flows within and out of the infected classes $\varsigma$ are defined by the vector $\mathbf{V}$, where these vectors are given by

$$
\mathbf{F}=\left(\begin{array}{c}
\frac{b_{1}^{\alpha} \beta_{1}^{\alpha} M_{s} B_{i}}{N_{B}} \\
\frac{b_{1}^{\alpha} \beta_{2}^{\alpha} M_{i} B_{s}}{N_{B}} \\
\frac{b_{2}^{\alpha} \beta_{3}^{\alpha} M_{i} S}{N_{B}} \\
0 \\
0 \\
0
\end{array}\right), \quad \mathbf{V}=\left(\begin{array}{c}
-\mu_{M}^{\alpha} M_{i} \\
-\left(d_{B}^{\alpha}+\Psi_{B}^{\alpha}+\mu_{B}^{\alpha}\right) B_{i} \\
-\left(\alpha_{w}^{\alpha}+\mu_{H}^{\alpha}\right) E \\
\alpha_{w}^{\alpha} E-\left(\gamma^{\alpha}+d_{I}^{\alpha}+r^{\alpha}+\mu_{H}^{\alpha}\right) I \\
\gamma^{\alpha} I-\left(d_{H}^{\alpha}+\tau^{\alpha}+\mu_{H}^{\alpha}\right) H \\
\tau^{\alpha} H+r^{\alpha} I-\mu_{H}^{\alpha} R
\end{array}\right) .
$$

Let us differentiate the above matrices $\mathbf{F}$ and $\mathbf{V}$ with respect to the infected variables $\zeta$ and evaluate at the disease-free equilibrium

$$
\mathbf{F}=\left(\begin{array}{ll}
f_{1} & f_{2} \\
f_{3} & f_{4}
\end{array}\right), \quad \mathbf{V}=\left(\begin{array}{ll}
v_{1} & v_{2} \\
v_{3} & v_{4}
\end{array}\right)
$$

where

$$
\begin{aligned}
& f_{1}=\left(\begin{array}{cc}
0 & \frac{b^{\alpha} \beta_{1}^{\alpha} M_{s}}{B_{s}+S} \\
\frac{b^{\alpha} \beta_{2}^{\alpha} B_{s}}{B_{s}+S} & 0
\end{array}\right), \quad f_{2}=\left(\begin{array}{cccc}
0 & 0 & 0 & 0 \\
0 & 0 & 0 & 0
\end{array}\right) \\
& f_{3}=\left(\begin{array}{cccc}
\frac{b^{\alpha} \beta_{3}^{\alpha} S}{B_{s}+S} & 0 & 0 & 0 \\
0 & 0 & 0 & 0
\end{array}\right)^{T}, \quad f_{4}=\left(\begin{array}{llll}
0 & 0 & 0 & 0 \\
0 & 0 & 0 & 0 \\
0 & 0 & 0 & 0 \\
0 & 0 & 0 & 0
\end{array}\right) \text {, } \\
& v_{1}=\left(\begin{array}{cc}
\mu_{M}^{\alpha} & 0 \\
0 & \mu_{B}^{\alpha}+\Psi_{B}^{\alpha}+d_{B}^{\alpha}
\end{array}\right), \quad v_{2}=\left(\begin{array}{cccc}
0 & 0 & 0 & 0 \\
0 & 0 & 0 & 0
\end{array}\right), \quad v_{3}=\left(\begin{array}{cccc}
0 & 0 & 0 & 0 \\
0 & 0 & 0 & 0
\end{array}\right)^{T} \\
& v_{4}=\left(\begin{array}{cccc}
\mu_{H}^{\alpha}+\alpha_{\omega}^{\alpha} & 0 & 0 & 0 \\
-\alpha_{\omega}^{\alpha} & \gamma^{\alpha}+d_{I}^{\alpha}+r^{\alpha}+\mu_{H}^{\alpha} & 0 & 0 \\
0 & -\gamma^{\alpha} & \tau^{\alpha}+\mu_{H}^{\alpha}+d_{H}^{\alpha} & 0 \\
0 & -r^{\alpha} & -\tau^{\alpha} & \mu_{H}^{\alpha}
\end{array}\right) \text {, }
\end{aligned}
$$

then the basic reproduction number $R_{0}$ for system (6) with spectral radius $\rho$ is defined by

$$
\begin{aligned}
R_{0} & =\rho\left(\mathbf{F V}^{-1}\right) \\
& =\frac{b^{\alpha} \sqrt{\mu_{M}^{\alpha}\left(\mu_{B}^{\alpha}+\Psi_{B}^{\alpha}+d_{B}^{\alpha}\right) \beta_{1}^{\alpha} \beta_{2}^{\alpha} \grave{M}_{s} \grave{B}_{s}}}{\mu_{M}^{\alpha}\left(\mu_{B}^{\alpha}+\Psi_{B}^{\alpha}+d_{B}^{\alpha}\right)\left(\grave{B}_{s}+\grave{S}\right)} .
\end{aligned}
$$

Lemma 4.1 ([61]) The disease-free equilibrium point $\varepsilon_{\mathrm{eq}}$ is locally asymptotically stable iff all eigenvalues $\lambda_{i}$ satisfy $\left|\arg \lambda_{i}\right|>\frac{\alpha \pi}{2}$. 
Proof To analyze the stability of the disease-free equilibrium (DFE) point, let us have that [62]

$$
\begin{array}{lcc}
{ }_{a}^{c} D_{t}^{\alpha} M_{s}=0, & { }_{a}^{c} D_{t}^{\alpha} M_{i}=0, & { }_{a}^{c} D_{t}^{\alpha} B_{s}=0, \\
{ }_{a}^{c} D_{t}^{\alpha} B_{i}=0, & { }_{a}^{c} D_{t}^{\alpha} S=0, & { }_{a}^{c} D_{t}^{\alpha} E=0, \\
{ }_{a}^{c} D_{t}^{\alpha} I=0, & { }_{a}^{c} D_{t}^{\alpha} H=0, & { }_{a}^{c} D_{t}^{\alpha} R=0 .
\end{array}
$$

Let us consider the coordinate transformation $m_{s}(t)=M_{s}(t)-\check{\check{M}}_{s}, m_{i}(t)=M_{i}(t)-\check{\check{M}}_{i}$,

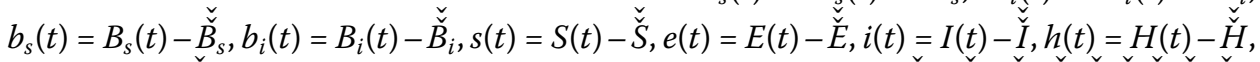

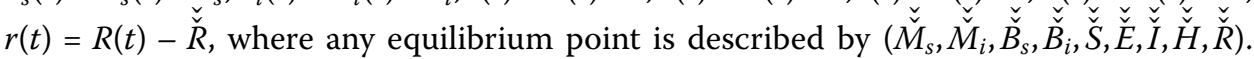
When $\check{M}_{i}=0, \check{\check{B}}_{i}=0$, and $\check{I}=0$, then the DFE point is $\varepsilon_{\text {eq }}=\left(\frac{\lambda_{M}^{\alpha}}{\mu_{M}^{\alpha}}, 0, \frac{\lambda_{B}^{\alpha}}{\Psi_{B}^{\alpha}+\mu_{B}^{\alpha}}, 0, \frac{\lambda_{H}^{\alpha}}{\mu_{H}^{\alpha}}, 0,0,0,0\right)$. The characteristic equation can be calculated at the DFE point. It can be written as follows:

$$
\begin{aligned}
& \left(J_{\varepsilon_{\mathrm{eq}}}-\lambda \mathcal{I}\right) \\
& \quad=\left(\begin{array}{ccccccccc}
-\mu_{M}^{\alpha}-\lambda & 0 & 0 & 0 & 0 & 0 & 0 & 0 & 0 \\
0 & -\mu_{M}^{\alpha}-\lambda & 0 & 0 & 0 & 0 & 0 & 0 & 0 \\
0 & -b_{1}^{\alpha} \beta_{2}^{\alpha} & -\mathbf{c}_{1}-\lambda & 0 & 0 & 0 & 0 & 0 & 0 \\
0 & b_{1}^{\alpha} \beta_{2}^{\alpha} & 0 & -\mathbf{c}_{2}-\lambda & 0 & 0 & 0 & 0 & 0 \\
0 & -b_{2}^{\alpha} \beta_{3}^{\alpha} e^{-\lambda \mathbf{d}} & 0 & 0 & -\mu_{H}^{\alpha}-\lambda & 0 & 0 & 0 & 0 \\
0 & b_{2}^{\alpha} \beta_{3}^{\alpha} e^{-\lambda \mathbf{d}} & 0 & 0 & 0 & -\mathbf{c}_{3}-\lambda & 0 & 0 & 0 \\
0 & 0 & 0 & 0 & 0 & \alpha_{\omega}^{\alpha} & -\mathbf{c}_{4}-\lambda & 0 & 0 \\
0 & 0 & 0 & 0 & 0 & 0 & \gamma^{\alpha} & -\mathbf{c}_{5}-\lambda-\tau^{\alpha} e^{-\lambda \mathbf{d}} & 0 \\
0 & 0 & 0 & 0 & 0 & 0 & r^{\alpha} & \tau^{\alpha} e^{-\lambda \mathbf{d}} & -\mu_{H}^{\alpha}-\lambda
\end{array}\right),
\end{aligned}
$$

where $\mathcal{I}$ is the unit matrix, $\mathbf{c}_{1}=\left(\Psi_{B}^{\alpha}+\mu_{B}^{\alpha}\right), \mathbf{c}_{2}=\left(d_{B}^{\alpha}+\Psi_{B}^{\alpha}+\mu_{B}^{\alpha}\right), \mathbf{c}_{3}=\left(\alpha_{\omega}^{\alpha}+\mu_{H}^{\alpha}\right), \mathbf{c}_{4}=\left(\gamma^{\alpha}+\right.$ $\left.d_{I}^{\alpha}+r^{\alpha}+\mu_{H}^{\alpha}\right)$, and $\mathbf{c}_{5}=\left(d_{H}^{\alpha}+\mu_{H}^{\alpha}\right)$. Thus the characteristic equation corresponding to the above matrix is [62]

$$
\begin{aligned}
& \left|J_{\varepsilon_{\mathrm{eq}}}-\lambda \mathcal{I}\right|=0, \\
& \left(\lambda+\mu_{H}^{\alpha}\right)^{2}\left(\lambda+\mu_{M}^{\alpha}\right)^{2}\left(\lambda+\alpha_{\omega}^{\alpha}+\mu_{H}^{\alpha}\right)\left(\lambda+\Psi_{B}^{\alpha}+\mu_{B}^{\alpha}\right)\left(\lambda+d_{B}^{\alpha}+\Psi_{B}^{\alpha}+\mu_{B}^{\alpha}\right) \\
& \quad \times\left(\lambda+d_{H}^{\alpha}+\mu_{H}^{\alpha}+\tau^{\alpha} e^{-\lambda \mathbf{d}}\right)\left(\lambda+d_{I}^{\alpha}+\gamma^{\alpha}+\mu_{H}^{\alpha}+r^{\alpha}\right)=0 .
\end{aligned}
$$

If we have $\mathbf{d}=0$, then the eigenvalues of the Jacobian matrix $J_{\varepsilon_{0}}$ at the DFE point $\varepsilon_{0}$ are $\lambda_{1,2}=-\mu_{H}^{\alpha}, \lambda_{3,4}=-\mu_{M}^{\alpha}, \lambda_{5}=-\left(\alpha_{\omega}^{\alpha}+\mu_{H}^{\alpha}\right), \lambda_{6}=-\left(\Psi_{B}^{\alpha}+\mu_{B}^{\alpha}\right), \lambda_{7}=-\left(d_{B}^{\alpha}+\Psi_{B}^{\alpha}+\mu_{B}^{\alpha}\right), \lambda_{8}=-\left(d_{H}^{\alpha}+\right.$ $\left.\mu_{H}^{\alpha}+\tau^{\alpha}\right)$, and $\lambda_{9}=-\left(d_{I}^{\alpha}+\gamma^{\alpha}+\mu_{H}^{\alpha}+r^{\alpha}\right)$. According to the Routh-Hurwitz theorem [35], these roots are negative or have negative real parts and the eigenvalues satisfy Matignon's conditions [63] defined by $\left|\arg \lambda_{i}\right|>\frac{\alpha \pi}{2}$. Thus, the DFE point $\varepsilon_{\text {eq }}$ is asymptotically stable. If we consider $\mathbf{d}>0$, then $\left(\lambda+d_{H}^{\alpha}+\mu_{H}^{\alpha}+\tau^{\alpha} e^{-\lambda \mathbf{d}}\right)$ has no pure imaginary roots for any value of the delay $\mathbf{d}$. Hence, all roots of the characteristic equation have negative real parts, and the DFE point $\varepsilon_{\text {eq }}$ is locally asymptotically stable according to Matignon's conditions [63] defined by $\left|\arg \lambda_{i}\right|>\frac{\alpha \pi}{2}$.

\section{Stability analysis of the endemic equilibrium}

In this section, the stability analysis of the endemic equilibrium point is introduced. If at least one of the infected variables is not zero, then system (6) has an endemic equilibrium. The endemic equilibrium point is $\varepsilon_{\text {end }}=(0.5908,2398.7049,3.6028,36.7141,0.4071$, $0.5679,573.7086,10.2296,39.7060)$. The list of parameter values is introduced in Table 3. 
The basic reproduction number is $R_{0}>1$. According to the linearized system of (6), we have the following form:

$$
\frac{d}{d t} \boldsymbol{\Phi}=\Delta_{1} \boldsymbol{\Phi}+\Delta_{2} \boldsymbol{\Phi}_{\mathbf{d}}
$$

where

$$
\begin{aligned}
& \boldsymbol{\Phi}=\left(m_{s}(t), m_{i}(t), b_{s}(t), b_{i}(t), s(t), e(t), i(t), h(t), r(t)\right)^{T}, \\
& \boldsymbol{\Phi}_{\mathbf{d}}=\left(m_{s}(t-\mathbf{d}), m_{i}(t-\mathbf{d}), b_{s}(t-\mathbf{d}), b_{i}(t-\mathbf{d}), s(t-\mathbf{d}), e(t-\mathbf{d}), i(t-\mathbf{d}),\right. \\
& h(t-\mathbf{d}), r(t-\mathbf{d}))^{T} \text {, } \\
& \Delta_{1}=\left(\begin{array}{ccccccccc}
-0.0823 & 0 & 0 & -0.0011 & 0 & 0 & 0 & 0 & 0 \\
0.0663 & -0.0160 & 0 & 0.0011 & 0 & 0 & 0 & 0 & 0 \\
0 & -0.0016 & -1.1343 & 0 & 0 & 0 & 0 & 0 & 0 \\
0 & 0.0016 & 1.0822 & -0.1121 & 0 & 0 & 0 & 0 & 0 \\
0 & 0 & 0 & 0 & -0.0050 & 0 & 0 & 0 & 0 \\
0 & 0 & 0 & 0 & 0 & -0.1050 & 0 & 0 & 0 \\
0 & 0 & 0 & 0 & 0 & 0.1000 & -0.0061 & 0 & 0 \\
0 & 0 & 0 & 0 & 0 & 0 & 0.0009 & -0.0050 & 0 \\
0 & 0 & 0 & 0 & 0 & 0 & 0.0002 & 0 & -0.0050
\end{array}\right) \text {, } \\
& \Delta_{2}=\left(\begin{array}{ccccccccc}
0 & 0 & 0 & 0 & 0 & 0 & 0 & 0 & 0 \\
0 & 0 & 0 & 0 & 0 & 0 & 0 & 0 & 0 \\
0 & 0 & 0 & 0 & 0 & 0 & 0 & 0 & 0 \\
0 & 0 & 0 & 0 & 0 & 0 & 0 & 0 & 0 \\
0 & -\frac{b_{2}^{\alpha} \beta_{3}^{\alpha} \check{S}}{N_{H}} & 0 & 0 & -\frac{b_{2}^{\alpha} \beta_{3}^{\alpha} \check{\underline{M}}_{i}}{N_{H}} & 0 & 0 & 0 & 0 \\
0 & \frac{b_{2}^{\alpha} \beta_{3}^{\alpha} \check{S}}{N_{H}} & 0 & 0 & \frac{b_{2}^{\alpha} \beta_{3}^{\alpha} \breve{M}_{i}}{N_{H}} & 0 & 0 & 0 & 0 \\
0 & 0 & 0 & 0 & 0 & 0 & 0 & 0 & 0 \\
0 & 0 & 0 & 0 & 0 & 0 & 0 & -\tau^{\alpha} & 0 \\
0 & 0 & 0 & 0 & 0 & 0 & 0 & -\tau^{\alpha} & 0
\end{array}\right) .
\end{aligned}
$$

Corresponding to these matrices, the characteristic equation $\left|\lambda \mathcal{I}-\Delta_{1}-e^{-\lambda \mathbf{d}} \Delta_{2}\right|=0$ is written as follows:

$$
\begin{aligned}
\lambda^{9} & +\left(1.4708+0.1582 e^{-\lambda \mathbf{d}}\right) \lambda^{8}+\left(0.4229+0.2139 e^{-\lambda \mathbf{d}}+0.0054 e^{-2 \lambda \mathbf{d}}\right) \lambda^{7} \\
& +\left(0.0490+0.0658 e^{-\lambda \mathbf{d}}+0.0079 e^{-2 \lambda \mathbf{d}}\right) \lambda^{6} \\
& +\left(0.0026+0.0074 e^{-\lambda \mathbf{d}}+0.0022 e^{-2 \lambda \mathbf{d}}\right) \lambda^{5} \\
& +\left(5.9414 \times 10^{-5}+3.6988 \times 10^{-4} e^{-\lambda \mathbf{d}}+2.4288 \times 10^{-4} e^{-2 \lambda \mathbf{d}}\right) \lambda^{4} \\
& +\left(6.7012 \times 10^{-7}+7.5511 \times 10^{-6} e^{-\lambda \mathbf{d}}+1.1435 \times 10^{-5} e^{-2 \lambda \mathbf{d}}\right) \lambda^{3} \\
& +\left(3.9229 \times 10^{-9}+6.8272 \times 10^{-8} e^{-\lambda \mathbf{d}}+2.0107 \times 10^{-7} e^{-2 \lambda \mathbf{d}}\right) \lambda^{2} \\
& +\left(1.1512 \times 10^{-11}+2.7933 \times 10^{-10} e^{-\lambda \mathbf{d}}+1.3295 \times 10^{-9} e^{-2 \lambda \mathbf{d}}\right) \lambda \\
& +\left(1.3423 \times 10^{-14}+4.2476 \times 10^{-13} e^{-\lambda \mathbf{d}}+2.9051 \times 10^{-12} e^{-2 \lambda \mathbf{d}}\right)=0 .
\end{aligned}
$$


If we have $\mathbf{d}=0$, then the characteristic equation is written in the following form:

$$
\begin{aligned}
\lambda^{9} & +1.6290 \lambda^{8}+0.6602 \lambda^{7}+0.1227 \lambda^{6}+0.0122 \lambda^{5}+6.7217 \times 10^{-4} \lambda^{4} \\
& +1.9656 \times 10^{-5} \lambda^{3}+2.7326 \times 10^{-7} \lambda^{2}+1.6203 \times 10^{-9} \lambda \\
& +3.3433 \times 10^{-12}=0 .
\end{aligned}
$$

The roots of the above equation are $\lambda_{1}=-0.0160, \lambda_{2}=-0.1121, \lambda_{3}=-0.1132, \lambda_{4}=$ $-0.1050, \lambda_{5}=-0.0823, \lambda_{6}=-1.1343, \lambda_{7}=-0.0050, \lambda_{8}=-0.0061, \lambda_{9}=-0.0550$. These roots are negative or have negative real parts and the eigenvalues satisfy Matignon's conditions [63] defined by $\left|\arg \lambda_{i}\right|>\frac{\alpha \pi}{2}$. Thus, the endemic equilibrium point $\varepsilon_{\text {end }}$ is asymptotically stable. If we consider $\mathbf{d}>0$, then these roots of the characteristic equation have no pure imaginary roots for any value of the delay $\mathbf{d}$. Thus, all roots of the characteristic equation have negative real parts according to Matignon's conditions [63] defined by $\left|\arg \lambda_{i}\right|>\frac{\alpha \pi}{2}$. Therefore, the endemic equilibrium is locally asymptotically stable.

\section{The optimal control problem}

In this section, we present the fractional optimal control in transmission dynamics of WNV model (6) with a time delay in the state variables $M_{i}(t), S(t)$, and $H(t)$. Two control functions are considered $u_{1}$ and $u_{2}$. The control $u_{1}$ represents the level of larvacide and adulticide used for mosquito control administered at mosquito breeding sites. The control $u_{2}$ measures the level of successful prevention (personal protection) efforts. Hence, we introduce a time delay $\mathbf{d}_{\mathbf{u}}$ in the control $u_{2}$ that represents the time required for the WNV prevention. The resulting model of WNV is given by the following system of nonlinear ordinary delay fractional-order differential equations:

$$
\begin{aligned}
& { }_{a}^{c} D_{t}^{\alpha} M_{s}(t)=\lambda_{M}^{\alpha} N_{M}\left(1-u_{1}(t)\right)-\frac{b_{1}^{\alpha} \beta_{1}^{\alpha} M_{s}(t) B_{i}(t)}{N_{B}(t)}-\mu_{M}^{\alpha} M_{s}(t)-r_{0}^{\alpha} u_{1}(t) M_{s}(t), \\
& { }_{a}^{c} D_{t}^{\alpha} M_{i}(t)=\frac{b_{1}^{\alpha} \beta_{1}^{\alpha} M_{s}(t) B_{i}(t)}{N_{B}(t)}-\mu_{M}^{\alpha} M_{i}(t)-r_{0}^{\alpha} u_{1}(t) M_{i}(t), \\
& { }_{a}^{c} D_{t}^{\alpha} B_{s}(t)=\lambda_{B}^{\alpha}+\rho^{\alpha} N_{B}(t)-\frac{b_{1}^{\alpha} \beta_{2}^{\alpha} M_{i}(t) B_{s}(t)}{N_{B}(t)}-\left(\Psi_{B}^{\alpha}+\mu_{B}^{\alpha}\right) B_{s}(t), \\
& { }_{a}^{c} D_{t}^{\alpha} B_{i}(t)=\frac{b_{1}^{\alpha} \beta_{2}^{\alpha} M_{i}(t) B_{s}(t)}{N_{B}(t)}-\left(d_{B}^{\alpha}+\Psi_{B}^{\alpha}+\mu_{B}^{\alpha}\right) B_{i}(t), \\
& { }_{a}^{c} D_{t}^{\alpha} S(t)=\lambda_{H}^{\alpha}+\gamma_{H}^{\alpha} N_{H}(t)-\frac{b_{2}^{\alpha} \beta_{3}^{\alpha} M_{i}(t-\mathbf{d}) S(t-\mathbf{d})\left(1-u_{2}\left(t-\mathbf{d}_{\mathbf{u}}\right)\right)}{N_{H}(t)}-\mu_{H}^{\alpha} S(t), \\
& { }_{a}^{c} D_{t}^{\alpha} E(t)=\frac{b_{2}^{\alpha} \beta_{3}^{\alpha} M_{i}(t-\mathbf{d}) S(t-\mathbf{d})\left(1-u_{2}\left(t-\mathbf{d}_{\mathbf{u}}\right)\right)}{N_{H}(t)}-\left(\alpha_{w}^{\alpha}+\mu_{H}^{\alpha}\right) E(t), \\
& { }_{a}^{c} D_{t}^{\alpha} I(t)=\alpha_{w}^{\alpha} E(t)-\left(\gamma^{\alpha}+d_{I}^{\alpha}+r^{\alpha}+\mu_{H}^{\alpha}\right) I(t), \\
& { }_{a}^{c} D_{t}^{\alpha} H(t)=\gamma^{\alpha} I(t)-\left(d_{H}^{\alpha}+\mu_{H}^{\alpha}\right) H(t)-\tau^{\alpha} H(t-\mathbf{d}), \\
& { }_{a}^{c} D_{t}^{\alpha} R(t)=\tau^{\alpha} H(t-\mathbf{d})+r^{\alpha} I(t)-\mu_{H}^{\alpha} R(t) .
\end{aligned}
$$

We consider that $\mathbf{d}_{\mathbf{u}}=\mathbf{d}$. Let us denote the state and control variables of the control system (16) by $\mathfrak{X}=\left(M_{s}, M_{i}, B_{s}, B_{i}, S, E, I, H, R\right) \in \mathbb{R}^{9}$ and $\mathfrak{U}=\left(u_{1}, u_{2}\right) \in \mathbb{R}^{2}$. We consider the 
following objective function which is quadratic in the control variable:

$$
J^{*}(\mathfrak{X}, \mathfrak{U})=\int_{0}^{T_{f}}\left(A_{1} E(t)+A_{2} I(t)+A_{3} N_{M}(t)+B_{1} u_{1}^{2}(t)+B_{2} u_{2}^{2}(t)\right) d t .
$$

The optimal control problem is defined by determining a control function $\mathfrak{U}=\left(u_{1}, u_{2}\right) \in$ $\mathbb{R}^{2}$ that minimizes the cost functional $J^{*}(\mathfrak{X}, \mathfrak{U})$ subject to the constraints $(16)$. To get the necessary optimality conditions of the optimal control problem described by time delay in state and control variables, see [64]. To get the Hamiltonian for the delayed fractional optimal control problem, let us introduce the delayed state variables $\mathfrak{C}_{2}=\mathfrak{X}_{2}(t-\mathbf{d})=M_{i}(t-$ d), $\mathfrak{C}_{5}=\mathfrak{X}_{5}(t-\mathbf{d})=S(t-\mathbf{d}), \mathfrak{C}_{8}=\mathfrak{X}_{8}(t-\mathbf{d})=H(t-\mathbf{d})$ and the delayed control variable $\mathfrak{A}_{2}=\mathfrak{U}_{2}(t-\mathbf{d})=u_{2}(t-\mathbf{d})$. The Hamiltonian for the objective functional (17) and the control system (16) is given by

$$
\begin{aligned}
\mathcal{H}^{*}( & \left.\mathfrak{X}, \mathfrak{C}_{2}, \mathfrak{C}_{5}, \mathfrak{C}_{8}, \lambda^{*}, u_{1}, \mathfrak{A}_{2}\right) \\
= & -\left(A_{1} E+A_{2} I+A_{3} N_{M}+B_{1} u_{1}^{2}+B_{2} u_{2}^{2}\right) \\
& +\lambda_{1}^{*}\left[\lambda_{M}^{\alpha} N_{M}\left(1-u_{1}\right)-\frac{b_{1}^{\alpha} \beta_{1}^{\alpha} M_{s} B_{i}}{N_{B}}-\mu_{M}^{\alpha} M_{s}-r_{0}^{\alpha} u_{1} M_{s}\right] \\
& +\lambda_{2}^{*}\left[\frac{b_{1}^{\alpha} \beta_{1}^{\alpha} M_{s} B_{i}}{N_{B}}-\mu_{M}^{\alpha} M_{i}-r_{0}^{\alpha} u_{1} M_{i}\right] \\
& +\lambda_{3}^{*}\left[\lambda_{B}^{\alpha}+\rho^{\alpha} N_{B}-\frac{b_{1}^{\alpha} \beta_{2}^{\alpha} M_{i} B_{s}}{N_{B}}-\left(\Psi_{B}^{\alpha}+\mu_{B}^{\alpha}\right) B_{s}\right] \\
& +\lambda_{4}^{*}\left[\frac{b_{1}^{\alpha} \beta_{2}^{\alpha} M_{i} B_{s}}{N_{B}}-\left(d_{B}^{\alpha}+\Psi_{B}^{\alpha}+\mu_{B}^{\alpha}\right) B_{i}\right] \\
& +\lambda_{5}^{*}\left[\lambda_{H}^{\alpha}+\gamma_{H}^{\alpha} N_{H}-\frac{b_{2}^{\alpha} \beta_{3}^{\alpha} \mathfrak{C}_{2} \mathfrak{C}_{5}\left(1-\mathfrak{A}_{2}\right)}{N_{H}}-\mu_{H}^{\alpha} S\right] \\
& +\lambda_{6}^{*}\left[\frac{b_{2}^{\alpha} \beta_{3}^{\alpha} \mathfrak{C}_{2} \mathfrak{C}_{5}\left(1-\mathfrak{A}_{2}\right)}{N_{H}}-\left(\alpha_{w}^{\alpha}+\mu_{H}^{\alpha}\right) E\right] \\
& +\lambda_{7}^{*}\left[\alpha_{w}^{\alpha} E-\left(\gamma^{\alpha}+d_{I}^{\alpha}+r^{\alpha}+\mu_{H}^{\alpha}\right) I\right] \\
& +\lambda_{8}^{*}\left[\gamma^{\alpha} I-\left(d_{H}^{\alpha}+\mu_{H}^{\alpha}\right) H-\tau^{\alpha} \mathfrak{C}_{8}\right] \\
& +\lambda_{9}^{*}\left[\tau^{\alpha} \mathfrak{C}_{8}+r^{\alpha} I-\mu_{H}^{\alpha} R\right],
\end{aligned}
$$

where the adjoint variable is $\lambda^{*}=\left(\lambda_{1}^{*}, \lambda_{2}^{*}, \lambda_{3}^{*}, \lambda_{4}^{*}, \lambda_{5}^{*}, \lambda_{6}^{*}, \lambda_{7}^{*}, \lambda_{8}^{*}, \lambda_{9}^{*}\right) \in \mathbb{R}^{9}$. The adjoint equations are given by

$$
\begin{aligned}
& { }_{t}^{c} D_{T_{f}}^{\alpha} \lambda_{1}^{*}(t)=-\frac{\partial \mathcal{H}^{*}(t)}{\partial M_{s}(t)}, \\
& { }_{t}^{c} D_{T_{f}}^{\alpha} \lambda_{2}^{*}(t)=-\frac{\partial \mathcal{H}^{*}(t)}{\partial M_{i}(t)}+\chi_{\left[0, T_{f}-\mathbf{d}\right]} \frac{\partial \mathcal{H}^{*}(t+\mathbf{d})}{\partial \mathfrak{C}_{2}}, \\
& { }_{t}^{c} D_{T_{f}}^{\alpha} \lambda_{3}^{*}(t)=-\frac{\partial \mathcal{H}^{*}(t)}{\partial B_{s}(t)}, \quad{ }_{t}^{c} D_{T_{f}}^{\alpha} \lambda_{4}^{*}(t)=-\frac{\partial \mathcal{H}^{*}(t)}{\partial B_{i}(t)}, \\
& { }_{t}^{c} D_{T_{f}}^{\alpha} \lambda_{5}^{*}(t)=-\frac{\partial \mathcal{H}^{*}(t)}{\partial S(t)}+\chi\left[0, T_{f}-\mathbf{d}\right] \frac{\partial \mathcal{H}^{*}(t+\mathbf{d})}{\partial \mathfrak{C}_{5}}, \\
& { }_{t}^{c} D_{T_{f}}^{\alpha} \lambda_{6}^{*}(t)=-\frac{\partial \mathcal{H}^{*}(t)}{\partial E(t)}, \quad{ }_{t}^{c} D_{T_{f}}^{\alpha} \lambda_{7}^{*}(t)=-\frac{\partial \mathcal{H}^{*}(t)}{\partial I(t)},
\end{aligned}
$$




$$
\begin{aligned}
& { }_{t}^{c} D_{T_{f}}^{\alpha} \lambda_{8}^{*}(t)=-\frac{\partial \mathcal{H}^{*}(t)}{\partial H(t)}+\chi_{\left[0, T_{f}-\mathbf{d}\right]} \frac{\partial \mathcal{H}^{*}(t+\mathbf{d})}{\partial \mathfrak{C}_{8}}, \\
& { }_{t}^{c} D_{T_{f}}^{\alpha} \lambda_{9}^{*}(t)=-\frac{\partial \mathcal{H}^{*}(t)}{\partial R(t)},
\end{aligned}
$$

where

$$
\chi_{\left[0, T_{f}-\mathbf{d}\right]}= \begin{cases}1, & \text { if } t \in\left[0, T_{f}-\mathbf{d}\right], \\ 0, & \text { otherwise. }\end{cases}
$$

The equations for $\lambda_{2}^{*}(t), \lambda_{5}^{*}(t)$, and $\lambda_{8}^{*}(t)$ contain the advanced time $t+\mathbf{d}$. Therefore, the Lagrange multipliers can be written as follows:

$$
\begin{aligned}
& { }_{t}^{c} D_{T_{f}}^{\alpha} \lambda_{1}^{*}=-A_{3}-\left(\lambda_{2}^{*}-\lambda_{1}^{*}\right) b \beta_{1} B_{i} \xi-\lambda_{1}^{*}\left[\lambda_{M}\left(1-u_{1}\right)-\left(\mu_{1}+\mu_{2} N_{M}\right)\right. \\
& \left.-\mu_{2} M_{s}-r_{0} u_{1}\right]+\lambda_{2}^{*} \mu_{2} M_{i} \\
& { }_{t}^{c} D_{T_{f}}^{\alpha} \lambda_{2}^{*}=-A_{3}-\lambda_{1}^{*}\left[\lambda_{M}\left(1-u_{1}\right)-\mu_{2} M_{s}\right]+\lambda_{2}^{*}\left[\mu_{1}+\mu_{2} N_{M}+\mu_{2} M_{i}+r_{0} u_{1}\right] \\
& -\left(\lambda_{4}^{*}-\lambda_{3}^{*}\right) b \beta_{2} B_{s} \xi-b \beta_{3} S\left(1-u_{2}\right) \xi\left(\lambda_{6}^{*}-\lambda_{5}^{*}\right) \\
& +\chi_{\left[0, T_{f}-\mathbf{d}\right]} \frac{b_{2}^{\alpha} \beta_{3}^{\alpha} \mathfrak{C}_{5}\left(1-\mathfrak{A}_{2}\right)}{N_{H}}\left(\lambda_{6}^{*}(t+\mathbf{d})-\lambda_{5}^{*}(t+\mathbf{d})\right), \\
& { }_{t}^{c} D_{T_{f}}^{\alpha} \lambda_{3}^{*}=\left(\lambda_{2}^{*}-\lambda_{1}^{*}\right) b \beta_{1} B_{i} M_{s} \xi^{2}-\lambda_{3}^{*}\left[\rho-b \beta_{2} M_{i}\left(\xi-B_{s} \xi^{2}\right)-\Psi_{B}-\mu_{B}\right] \\
& -\lambda_{4}^{*} b \beta_{2} M_{i}\left(\xi-B_{s} \xi^{2}\right)-\left(\lambda_{5}^{*}-\lambda_{6}^{*}\right) b \beta_{3} M_{i} S\left(1-u_{2}\right) \xi^{2}, \\
& { }_{t}^{c} D_{T_{f}}^{\alpha} \lambda_{4}^{*}=-\left(\lambda_{2}^{*}-\lambda_{1}^{*}\right) b \beta_{1} M_{s}\left(\xi-B_{i} \xi^{2}\right)-\lambda_{3}^{*}\left[\rho+b \beta_{2} M_{i} B_{s} \xi^{2}\right] \\
& +\lambda_{4}^{*}\left[b \beta_{2} M_{i} B_{S} \xi^{2}+\left(d_{B}+\Psi_{B}+\mu_{B}\right)\right]-\left(\lambda_{5}^{*}-\lambda_{6}^{*}\right) b \beta_{3} M_{i} S\left(1-u_{2}\right) \xi^{2}, \\
& { }_{t}^{c} D_{T_{f}}^{\alpha} \lambda_{5}^{*}=-\left(\lambda_{1}^{*}-\lambda_{2}^{*}\right) b \beta_{1} B_{i} M_{s} \xi^{2}-\left(\lambda_{3}^{*}-\lambda_{4}^{*}\right) b \beta_{2} M_{i} B_{s} \xi^{2} \\
& -\lambda_{5}^{*}\left[\gamma_{H}-b \beta_{3} M_{i}\left(1-u_{2}\right)\left(\xi-S \xi^{2}\right)-\mu_{4} S-\left(\mu_{3}+\mu_{4} N_{H}\right)\right] \\
& -\lambda_{6}^{*}\left[b \beta_{3} M_{i}\left(1-u_{2}\right)\left(\xi-S \xi^{2}\right)-\mu_{4} E\right]+\lambda_{7}^{*} \mu_{4} I+\lambda_{8}^{*} \mu_{4} H+\lambda_{9}^{*} \mu_{4} R \\
& +\chi_{\left[0, T_{f}-\mathbf{d}\right]} \frac{b_{2}^{\alpha} \beta_{3}^{\alpha} \mathfrak{C}_{2}\left(1-\mathfrak{A}_{2}\right)}{N_{H}}\left(\lambda_{6}^{*}(t+\mathbf{d})-\lambda_{5}^{*}(t+\mathbf{d})\right), \\
& { }_{t}^{c} D_{T_{f}}^{\alpha} \lambda_{6}^{*}=-A_{1}-\left(\lambda_{1}^{*}-\lambda_{2}^{*}\right) b \beta_{1} B_{i} M_{s} \xi^{2}-\left(\lambda_{3}^{*}-\lambda_{4}^{*}\right) b \beta_{2} M_{i} B_{s} \xi^{2} \\
& -\lambda_{5}^{*}\left[\gamma_{H}+b \beta_{3} M_{i} B_{S}\left(1-u_{2}\right) \xi^{2}-\mu_{4} S\right] \\
& +\lambda_{6}^{*}\left[b \beta_{3} M_{i} S\left(1-u_{2}\right) \xi^{2}+\alpha_{w}+\mu_{4} E+\mu_{3}+\mu_{4} N_{H}\right] \\
& -\lambda_{7}^{*}\left[\alpha_{w}-\mu_{4} I\right]+\lambda_{8}^{*} \mu_{4} H+\lambda_{9}^{*} \mu_{4} R, \\
& { }_{t}^{c} D_{T_{f}}^{\alpha} \lambda_{7}^{*}=-A_{2}-\left(\lambda_{1}^{*}-\lambda_{2}^{*}\right) b \beta_{1} B_{i} M_{s} \xi^{2}-\left(\lambda_{3}^{*}-\lambda_{4}^{*}\right) b \beta_{2} M_{i} B_{s} \xi^{2} \\
& -\lambda_{5}^{*}\left[\gamma_{H}+b \beta_{3} M_{i} S\left(1-u_{2}\right) \xi^{2}-\mu_{4} S\right]+\lambda_{6}^{*}\left[b \beta_{3} M_{i} S\left(1-u_{2}\right) \xi^{2}+\mu_{4} E\right] \\
& +\lambda_{7}^{*}\left[\gamma+d_{I}+r+\mu_{4} I+\mu_{3}+\mu_{4} N_{H}\right]-\lambda_{8}^{*}\left[\gamma-\mu_{4} H\right]-\lambda_{9}^{*}\left[r-\mu_{4} R\right], \\
& { }_{t}^{c} D_{T_{f}}^{\alpha} \lambda_{8}^{*}=-\left(\lambda_{1}^{*}-\lambda_{2}^{*}\right) b \beta_{1} M_{s} B_{i} \xi^{2}-\left(\lambda_{3}^{*}-\lambda_{4}^{*}\right) b \beta_{2} M_{i} B_{s} \xi^{2} \\
& -\lambda_{5}^{*}\left[\gamma_{H}+b \beta_{3} M_{i} S\left(1-u_{2}\right) \xi^{2}-\mu_{4} S\right]+\lambda_{6}^{*}\left[b \beta_{3} M_{i} S\left(1-u_{2}\right) \xi^{2}+\mu_{4} E\right]
\end{aligned}
$$




$$
\begin{aligned}
& +\lambda_{7}^{*} \mu_{4} I+\lambda_{8}^{*}\left[d_{H}+\tau+\mu_{4} N_{H}+\mu_{3}+\mu_{4} H\right]-\lambda_{9}^{*}\left[\tau-\mu_{4} R\right] \\
& +\chi_{\left[0, T_{f}-\mathbf{d}\right]} \tau^{\alpha}\left(\lambda_{9}^{*}(t+\mathbf{d})-\lambda_{8}^{*}(t+\mathbf{d})\right), \\
{ }_{t}^{c} D_{T_{f}}^{\alpha} \lambda_{9}^{*}= & -\left(\lambda_{1}^{*}-\lambda_{2}^{*}\right) b \beta_{1} M_{s} B_{i} \xi^{2}-\left(\lambda_{3}^{*}-\lambda_{4}^{*}\right) b \beta_{2} M_{i} B_{s} \xi^{2} \\
& -\lambda_{5}^{*}\left[\gamma_{H}+b \beta_{3} M_{i} S\left(1-u_{2}\right) \xi^{2}-\mu_{4} S\right]+\lambda_{6}^{*}\left[b \beta_{3} M_{i} S\left(1-u_{2}\right) \xi^{2}+\mu_{4} E\right] \\
& +\lambda_{7}^{*} \mu_{4} I+\lambda_{8}^{*} \mu_{4} H+\lambda_{9}^{*}\left[\mu_{4} R+\mu_{3}+\mu_{4} N_{H}\right] .
\end{aligned}
$$

Thus, the terminal state is free, the transversality conditions are

$$
\lambda_{i}^{*}\left(T_{f}\right)=0, \quad \forall i=1,2, \ldots, 9,
$$

and the characterization of the control variable $u_{1}$ is

$$
\frac{\partial \mathcal{H}^{*}(t)}{\partial u_{1}(t)}=0
$$

To characterize the control variable $u_{2}$, we introduce the following switching function:

$$
\begin{aligned}
\partial_{2}(t) & =\frac{\partial \mathcal{H}^{*}(t)}{\partial u_{2}(t)}+\chi_{\left[0, T_{f}-\mathbf{d}\right]} \frac{\partial \mathcal{H}^{*}(t+\mathbf{d})}{\partial \mathfrak{A}_{2}} \\
& = \begin{cases}\frac{1}{2} B_{2} u_{2}+\frac{b_{2}^{\alpha} \beta_{3}^{\alpha} \mathfrak{C}_{2} \mathfrak{C}_{5}\left(\lambda_{5}^{*}-\lambda_{6}^{*}\right)}{N_{H}}, & \text { for } 0 \leq t \leq T_{f}-\mathbf{d}, \\
\frac{1}{2} B_{2} u_{2}, & \text { for } T_{f}-\mathbf{d} \leq t \leq T_{f} .\end{cases}
\end{aligned}
$$

Thus, the maximum condition for the control variable $u_{2}(t)$ is equivalent to the maximization $\partial_{2}(t) u_{2}(t)=\max _{0 \leq u_{2} \leq 1} \partial_{2}(t) u_{2}(t)$.

\section{NSFD for fractional time-delay West Nile virus model}

In this section, the discretization of fractional delay WNV model is presented. We apply the NSFD method with Grünwald-Letnikov definition, so the discretization can be written as follows:

$$
\begin{aligned}
& \sum_{\iota=0}^{n+1} \omega_{\iota}^{\alpha} M_{s}^{n+1-\iota}=\lambda_{M}^{\alpha} N_{M}^{n}\left(1-u_{1}^{n}\right)-\frac{b_{1}^{\alpha} \beta_{1}^{\alpha} M_{s}^{n} B_{i}^{n}}{N_{B}^{n}}-\mu_{M}^{\alpha} M_{s}^{n}-r_{0}^{\alpha} u_{1}^{n} M_{s}^{n}, \\
& \sum_{\iota=0}^{n+1} \omega_{\iota}^{\alpha} M_{i}^{n+1-\iota}=\frac{b_{1}^{\alpha} \beta_{1}^{\alpha} M_{s}^{n} B_{i}^{n}}{N_{B}^{n}}-\mu_{M}^{\alpha} M_{i}^{n}-r_{0}^{\alpha} u_{1}^{n} M_{i}^{n}, \\
& \sum_{\iota=0}^{n+1} \omega_{\iota}^{\alpha} B_{s}^{n+1-\iota}=\lambda_{B}^{\alpha}+\rho^{\alpha} N_{B}^{n}-\frac{b_{1}^{\alpha} \beta_{2}^{\alpha} M_{i}^{n} B_{s}^{n}}{N_{B}^{n}}-\left(\Psi_{B}^{\alpha}+\mu_{B}^{\alpha}\right) B_{s}^{n}, \\
& \sum_{\iota=0}^{n+1} \omega_{\iota}^{\alpha} B_{i}^{n+1-\iota}=\frac{b_{1}^{\alpha} \beta_{2}^{\alpha} M_{i}^{n} B_{s}^{n}}{N_{B}^{n}}-\left(d_{B}^{\alpha}+\Psi_{B}^{\alpha}+\mu_{B}^{\alpha}\right) B_{i}^{n}, \\
& \sum_{\iota=0}^{n+1} \omega_{\iota}^{\alpha} S^{n+1-\iota}=\lambda_{H}^{\alpha}+\gamma_{H}^{\alpha} N_{H}^{n}-\frac{b_{2}^{\alpha} \beta_{3}^{\alpha} M_{i}^{n-\varrho} S^{n-\varrho}\left(1-u_{2}^{n-\varrho}\right)}{N_{H}^{n}}-\mu_{H}^{\alpha} S^{n}, \\
& \sum_{\iota=0}^{n+1} \omega_{\iota}^{\alpha} E^{n+1-\iota}=\frac{b_{2}^{\alpha} \beta_{3}^{\alpha} M_{i}^{n-\varrho} S^{n-\varrho}\left(1-u_{2}^{n-\varrho}\right)}{N_{H}^{n}}-\left(\alpha_{w}^{\alpha}+\mu_{H}^{\alpha}\right) E^{n},
\end{aligned}
$$



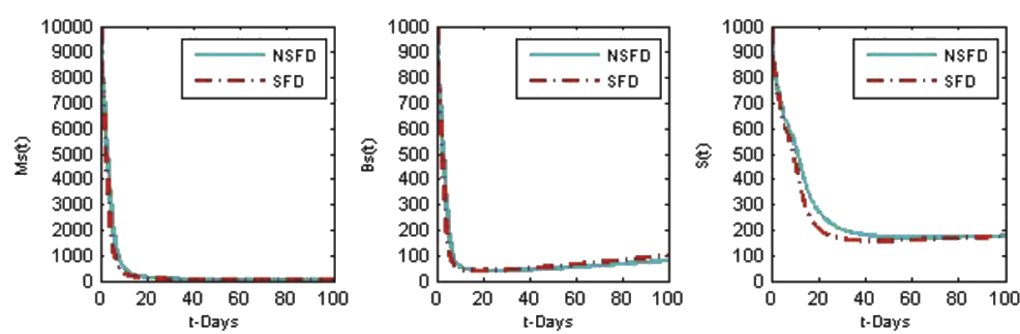

(a) Susceptibles
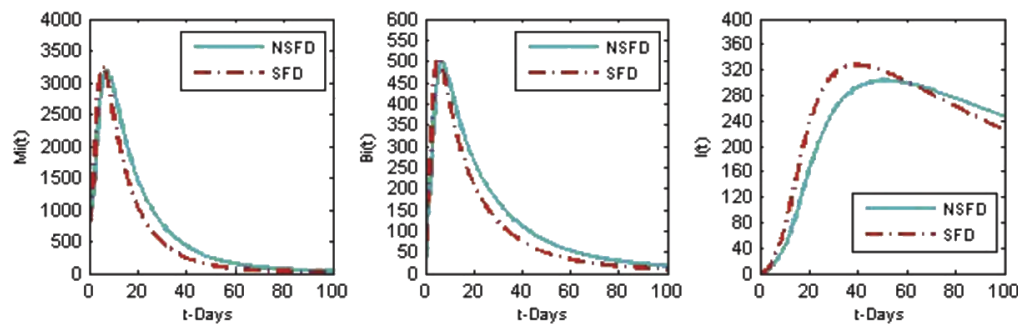

(b) Infected
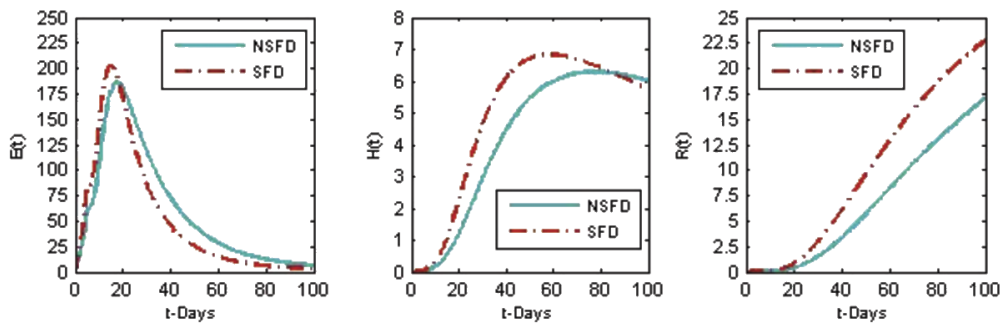

(c) Exposed, Hospitalized and Recovered Humans
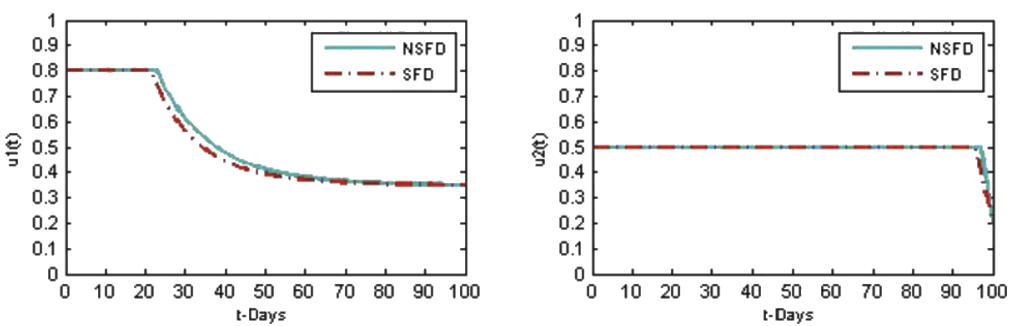

(d) Control Functions

Figure 1 The approximate solutions of the fractional delay optimal control problem by using NSFDM and SFDM. When $0 \leq u_{1} \leq 0.8,0 \leq u_{2} \leq 0.5$, with $\mathbf{d}=5$ and $\alpha=0.90$, and $\Delta t=0.5$

$$
\begin{aligned}
& \sum_{\iota=0}^{n+1} \omega_{\iota}^{\alpha} I^{n+1-\iota}=\alpha_{w}^{\alpha} E^{n}-\left(\gamma^{\alpha}+d_{I}^{\alpha}+r^{\alpha}+\mu_{H}^{\alpha}\right) I^{n}, \\
& \sum_{\iota=0}^{n+1} \omega_{\iota}^{\alpha} H^{n+1-\iota}=\gamma^{\alpha} I^{n}-\left(d_{H}^{\alpha}+\mu_{H}^{\alpha}\right) H^{n}-\tau^{\alpha} H^{n-\varrho}, \\
& \sum_{\iota=0}^{n+1} \omega_{\iota}^{\alpha} R^{n+1-\iota}=\tau^{\alpha} H^{n-\varrho}+r^{\alpha} I^{n}-\mu_{H}^{\alpha} R^{n},
\end{aligned}
$$

where $\omega_{0}^{\alpha}=(\varphi(\Delta t))^{-\alpha}, n=-\varrho,-\varrho+1, \ldots, 0,1$, and the local approximations are used for the nonlinear terms. We use the denominator function of the form $\varphi(\Delta t)=1-e^{-\Delta t}$. Thus, 

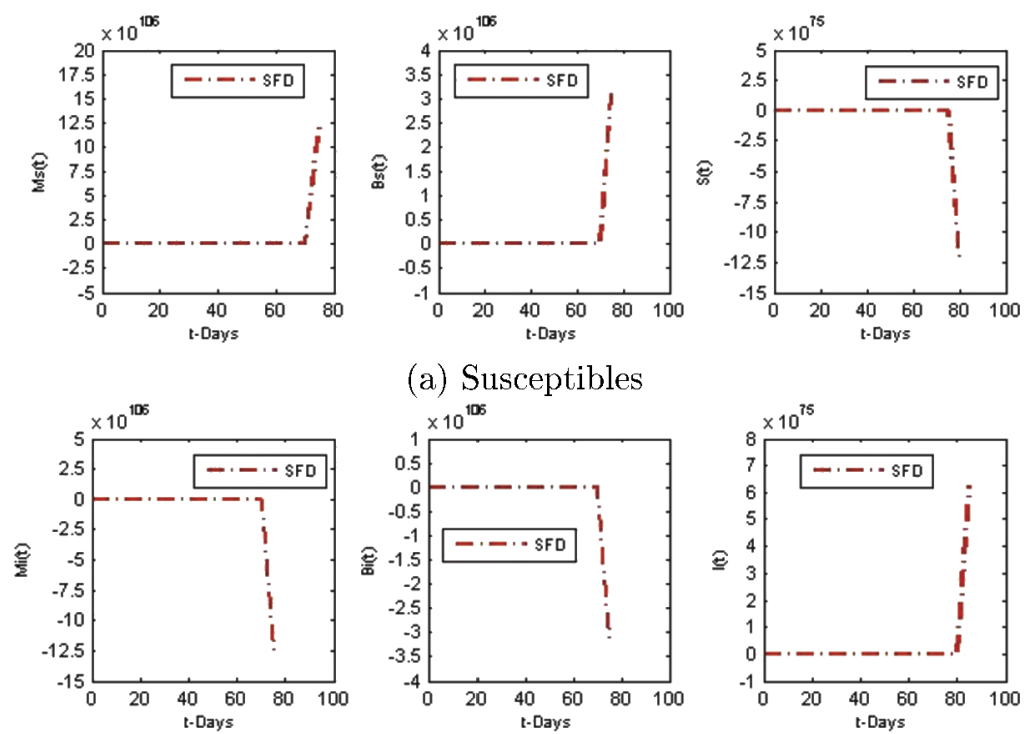

(a) Susceptibles
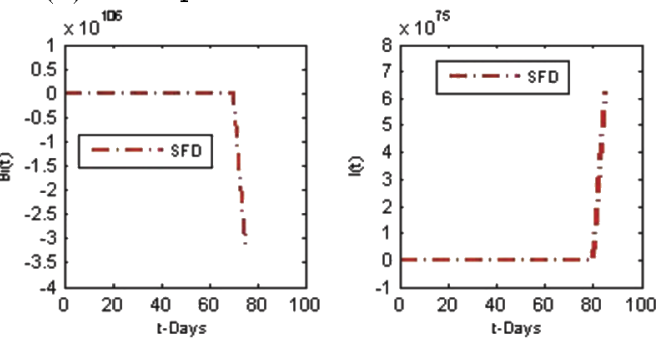

(b) Infected
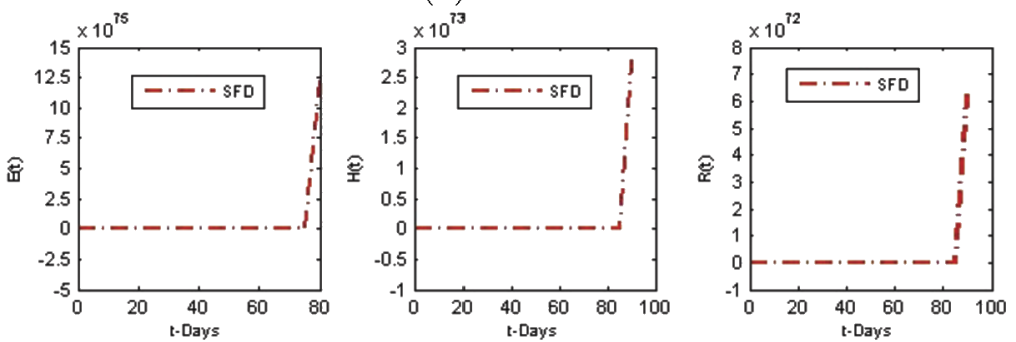

(c) Exposed, Hospitalized and Recovered Humans

Figure 2 The approximate solutions of the fractional delay optimal control problem by using SFDM. When $0 \leq u_{1} \leq 0.8,0 \leq u_{2} \leq 0.5$, with $\mathbf{d}=10$ and $\alpha=1$, and $\Delta t=5$

the discretized system (24) can be written as follows:

$$
\begin{aligned}
& M_{s}^{n+1}=\frac{\lambda_{M}^{\alpha} N_{M}^{n}\left(1-u_{1}^{n}\right)-\frac{b_{1}^{\alpha} \beta_{1}^{\alpha} M_{s}^{n} B_{i}^{n}}{N_{B}^{n}}-\mu_{M}^{\alpha} M_{s}^{n}-r_{0}^{\alpha} u_{1}^{n} M_{s}^{n}-\sum_{\iota=1}^{n+1} \omega_{\iota}^{\alpha} M_{s}^{n+1-\iota}}{\omega_{0}^{\alpha}}, \\
& M_{i}^{n+1}=\frac{\frac{b_{1}^{\alpha} \beta_{1}^{\alpha} M_{s}^{n} B_{i}^{n}}{N_{B}^{n}}-\mu_{M}^{\alpha} M_{i}^{n}-r_{0}^{\alpha} u_{1}^{n} M_{i}^{n}-\sum_{\iota=1}^{n+1} \omega_{\iota}^{\alpha} M_{i}^{n+1-\iota}}{\omega_{0}^{\alpha}}, \\
& B_{s}^{n+1}=\frac{\lambda_{B}^{\alpha}+\rho^{\alpha} N_{B}^{n}-\frac{b_{1}^{\alpha} \beta_{2}^{\alpha} M_{i}^{n} B_{s}^{n}}{N_{B}^{n}}-\left(\Psi_{B}^{\alpha}+\mu_{B}^{\alpha}\right) B_{s}^{n}-\sum_{\iota=1}^{n+1} \omega_{\iota}^{\alpha} B_{s}^{n+1-\iota}}{\omega_{0}^{\alpha}}, \\
& B_{i}^{n+1}=\frac{\frac{b_{1}^{\alpha} \beta_{2}^{\alpha} M_{i}^{n} B_{s}^{n}}{N_{B}^{n}}-\left(d_{B}^{\alpha}+\Psi_{B}^{\alpha}+\mu_{B}^{\alpha}\right) B_{i}^{n}-\sum_{l=1}^{n+1} \omega_{l}^{\alpha} B_{i}^{n+1-\iota}}{\omega_{0}^{\alpha}}, \\
& S^{n+1}=\frac{\lambda_{H}^{\alpha}+\gamma_{H}^{\alpha} N_{H}^{n}-\frac{b_{2}^{\alpha} \beta_{3}^{\alpha} M_{i}^{n-\varrho} S^{n-\varrho}\left(1-u_{2}^{n-\varrho}\right)}{N_{H}^{n}}-\mu_{H}^{\alpha} S^{n}-\sum_{\iota=1}^{n+1} \omega_{\iota}^{\alpha} S^{n+1-\iota}}{\omega_{0}^{\alpha}}, \\
& E^{n+1}=\frac{\frac{b_{2}^{\alpha} \beta_{3}^{\alpha} M_{i}^{n-\varrho} S^{n-\varrho}\left(1-u_{2}^{n-\varrho}\right)}{N_{H}^{n}}-\left(\alpha_{w}^{\alpha}+\mu_{H}^{\alpha}\right) E^{n}-\sum_{\iota=1}^{n+1} \omega_{\iota}^{\alpha} E^{n+1-\iota}}{\omega_{0}^{\alpha}},
\end{aligned}
$$



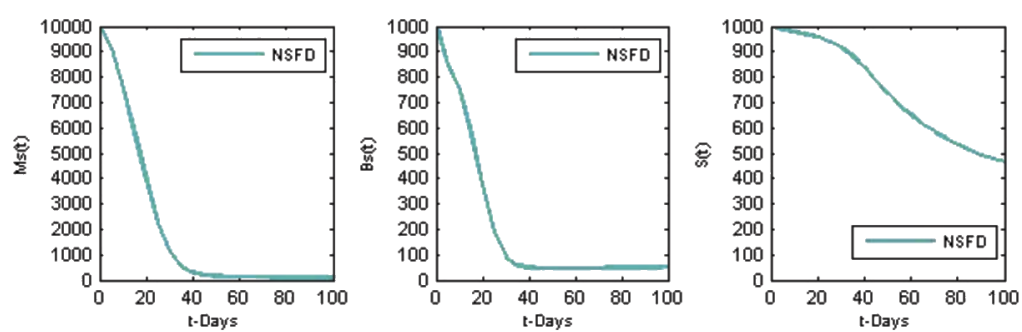

(a) Susceptibles
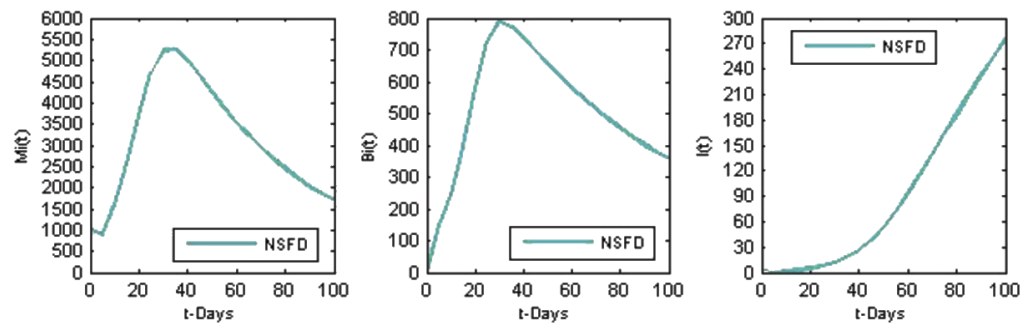

(b) Infected
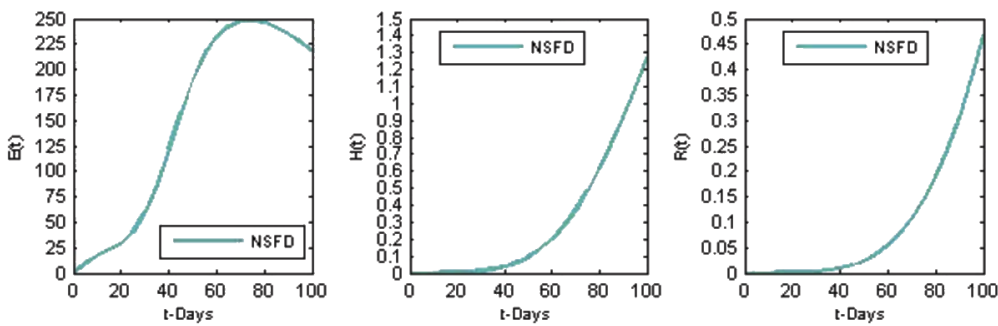

(c) Exposed, Hospitalized and Recovered Humans
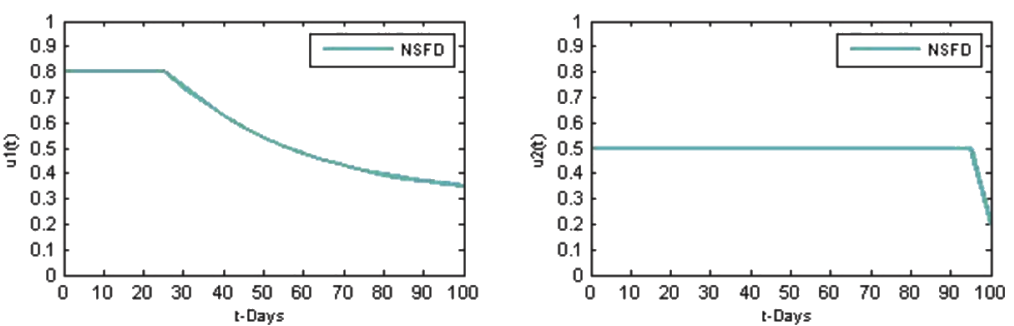

(d) Control Functions

Figure 3 The approximate solutions of the fractional delay optimal control problem by using NSFDM. When $0 \leq u_{1} \leq 0.8,0 \leq u_{2} \leq 0.5$, with $\mathbf{d}=10$ and $\alpha=1$, and $\Delta t=5$

$$
\begin{aligned}
& I^{n+1}=\frac{\alpha_{w}^{\alpha} E^{n}-\left(\gamma^{\alpha}+d_{I}^{\alpha}+r^{\alpha}+\mu_{H}^{\alpha}\right) I^{n}-\sum_{l=1}^{n+1} \omega_{l}^{\alpha} I^{n+1-\iota}}{\omega_{0}^{\alpha}}, \\
& H^{n+1}=\frac{\gamma^{\alpha} I^{n}-\left(d_{H}^{\alpha}+\mu_{H}^{\alpha}\right) H^{n}-\tau^{\alpha} H^{n-\varrho}-\sum_{l=1}^{n+1} \omega_{\iota}^{\alpha} H^{n+1-\iota}}{\omega_{0}^{\alpha}}, \\
& R^{n+1}=\frac{\tau^{\alpha} H^{n-\varrho}+r^{\alpha} I^{n}-\mu_{H}^{\alpha} R^{n}-\sum_{l=1}^{n+1} \omega_{l}^{\alpha} R^{n+1-\iota}}{\omega_{0}^{\alpha}}
\end{aligned}
$$

where

$$
N_{M}^{n}=M_{s}^{n}+M_{i}^{n},
$$



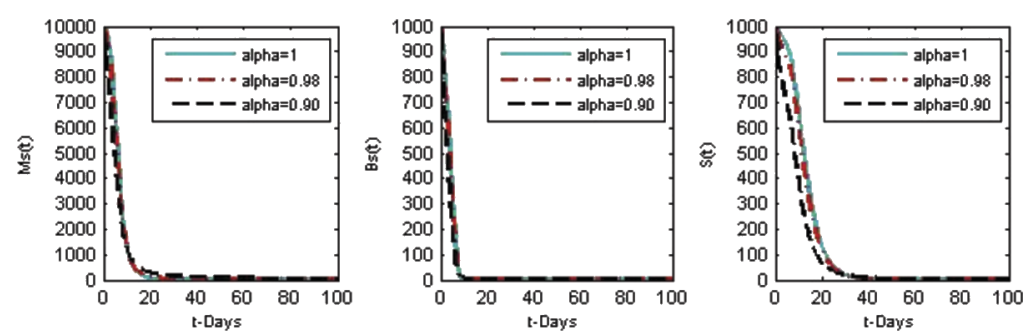

(a) Susceptibles
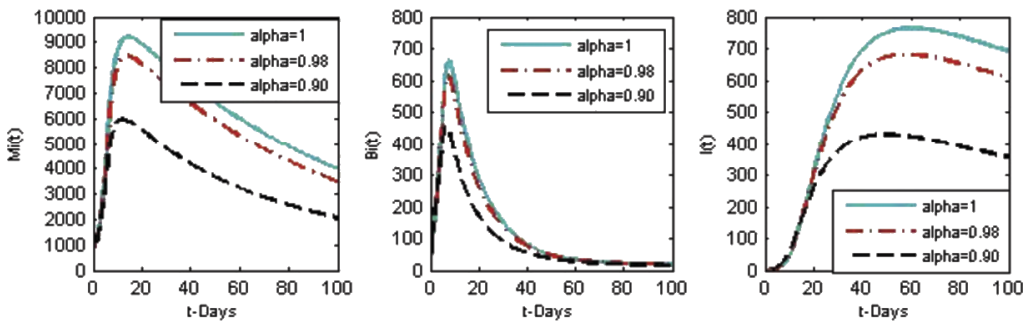

(b) Infected
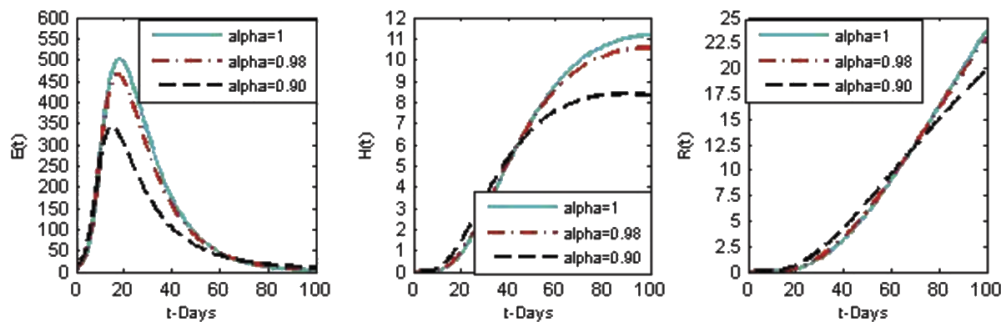

(c) Exposed, Hospitalized and Recovered Humans

Figure 4 The approximate solutions of the fractional delay WNV model without control (i.e., $u_{1}=u_{2}=0$ ) by using NSFDM. With $\mathbf{d}=1$ at different $\alpha$, and $\Delta t=1$

$$
\begin{aligned}
& N_{B}^{n}=B_{s}^{n}+B_{i}^{n}, \\
& N_{H}^{n}=S^{n}+E^{n}+I^{n}+H^{n}+R^{n} .
\end{aligned}
$$

\section{Numerical experiment}

Throughout this section for simulations, we use NSFDM and SFDM. These simulations are performed with initial conditions $\left(M_{s_{0}}, M_{i_{0}}, B_{s_{0}}, B_{i_{0}}, S_{0}, E_{0}, I_{0}, R_{0}, H_{0}\right)=(10,000,1000$, $1000,0,1000,0,0,0,0)$ and transversality conditions $\lambda_{i}\left(T_{f}\right)=0, \forall i=1,2, \ldots, 9$. For Figs. 19, the weights $A_{1}=A_{2}=1, A_{3}=10^{-4}$ in the cost functional (17), (i.e., the minimization of the number of exposed and infected humans is given more importance than the reduction of the total number of mosquitoes). Respectively, we use the upper bound of 0.8 and 0.5 on $u_{1}$ and $u_{2}$. All parameters are collected and given in Table 3. In Table 4, the sum of numerical values of the state variables $E\left(T_{f}\right)+I\left(T_{f}\right)$ at $T_{f}=100$ and the objective function $J^{*}(\mathfrak{X}, \mathfrak{U})$ in two cases, with and without control at different values of time delay $\mathbf{d}$ and different values of $\alpha$ with $\Delta t=0.1$, obtained by NSFD method, are given. According to comparisons that are presented in Table 4, it can be observed that, for example, the cost functional $J^{*}(\mathfrak{X}, \mathfrak{U})$ with control is $44,507,56,867$ at $\mathbf{d}=1$ and $\mathbf{d}=7$, respectively, in case of integer order and $J^{*}(\mathfrak{X}, \mathfrak{U})$ without control is $68,771,71,011$ at the same values of $\mathbf{d}$. But in case of fractional order $J^{*}(\mathfrak{X}, \mathfrak{U})$ with control is $38,732,49,617$ and $J^{*}(\mathfrak{X}, \mathfrak{U})$ without 

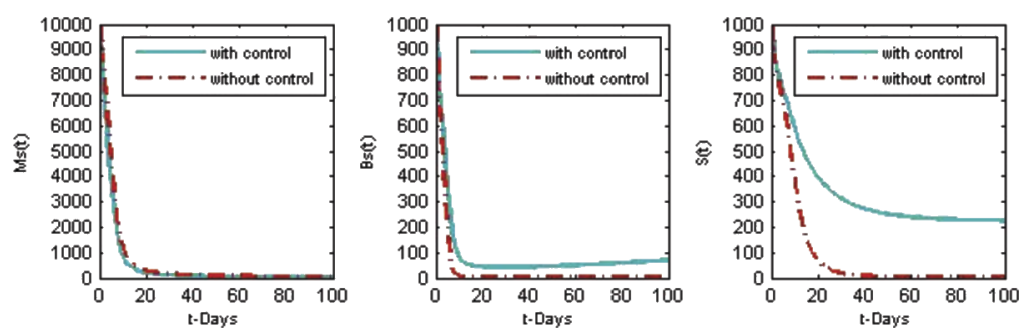

(a) Susceptibles
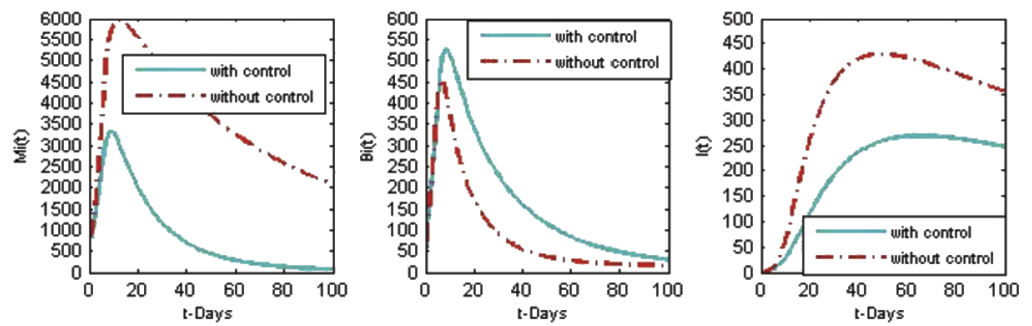

(b) Infected
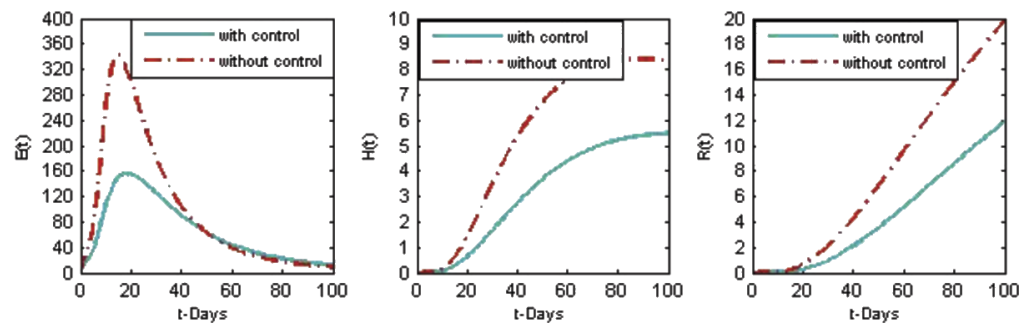

(c) Exposed, Hospitalized and Recovered Humans

Figure 5 The approximate solutions of the fractional delay optimal control problem by using NSFDM. When $0 \leq u_{1} \leq 0.8,0 \leq u_{2} \leq 0.5$, and without control (i.e., $u_{1}=u_{2}=0$ ) with $\mathbf{d}=1$ at $\alpha=0.90$, and $\Delta t=1$

control is 59,450, 61,304 for $\alpha=0.98$ and 21,901, 28,350 in control case but in case of without control 32,911, 34,564 for $\alpha=0.90$ at $\mathbf{d}=1$ and $\mathbf{d}=7$, respectively.

The main goal in this study is to reduce the infected and exposed human populations from the increase due to delay in treatment. Through the results obtained in Table 4, it can be noted that, if the time delay $\mathbf{d}$ is increased, the infected and exposed human populations are increased at different values of $\alpha$. For example, in case of non-delay optimal control problem at $\mathbf{d}=0$, it can be seen that the infected and exposed human populations are increased if the value of $\alpha$ is increased, and the better results occur at $\alpha=0.70$. While in the case of delay optimal control problem, it can be noted that at $\mathbf{d}=1$, the lowest value of the infected and exposed human populations occurs at $\alpha=0.70$, similarly for $\mathbf{d}=3,5,7$.

In Table 5, comparisons between NSFDM and SFDM at different values of time delay $\mathbf{d}$ and different values of $\alpha$ with $\Delta t=1$ at $T_{f}=100$ are presented. It can be seen that the values of the cost function $J^{*}(\mathfrak{X}, \mathfrak{U})$ are 44,560,62,325 at $\mathbf{d}=3$ and $\mathbf{d}=10$, respectively, using NSFDM, in case of integer order. But it takes the values 45,647, 62,325 at the same time delay $\mathbf{d}$ using SFDM. In case of fractional order, the values of $J^{*}(\mathfrak{X}, \mathfrak{U})$ are 40,721, 27,976 using NSFDM and 56,813, 38,610 using SFDM for $\alpha=0.98, \alpha=0.90$ at the same time delay $\mathbf{d}$. Thus, the objective function $J^{*}(\mathfrak{X}, \mathfrak{U})$ obtained using NSFDM is better than that obtained using SFDM. It can be concluded that the numerical treatments for the effec- 

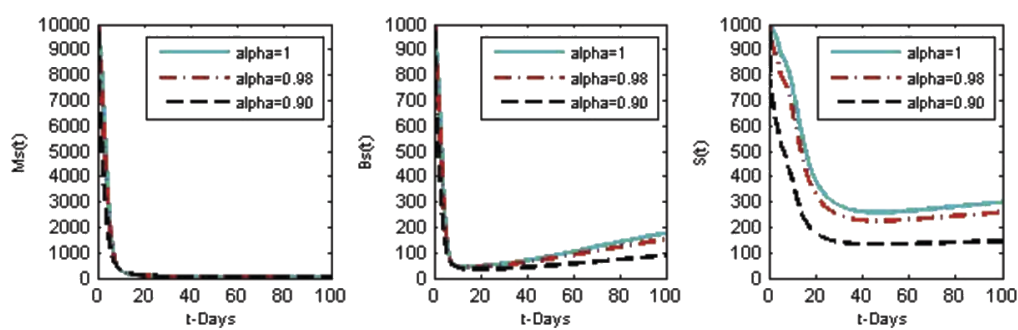

(a) Susceptibles
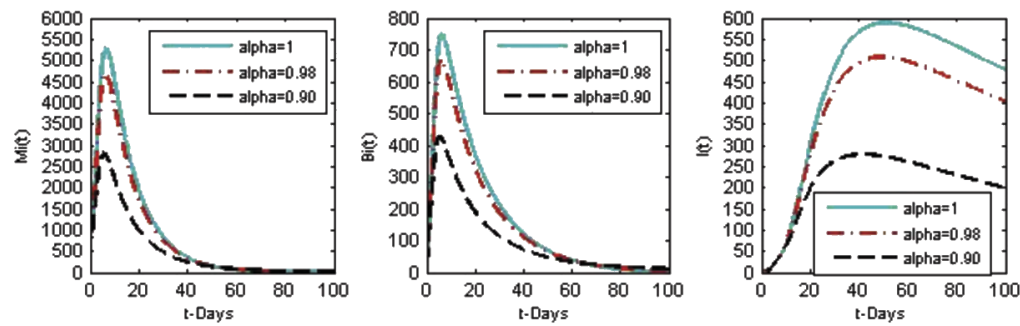

(b) Infected
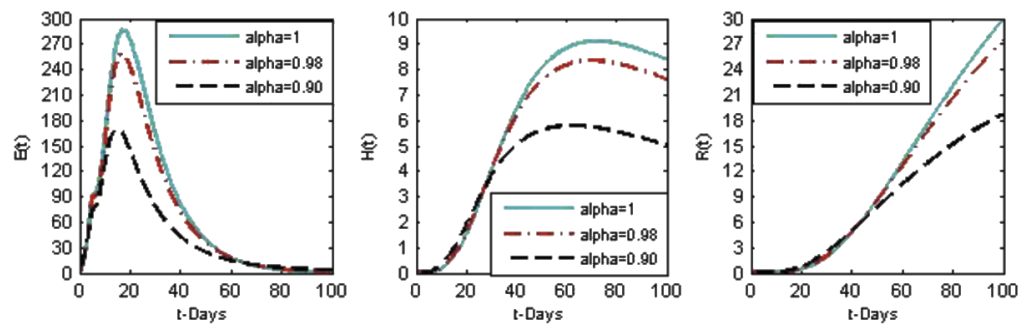

(c) Exposed, Hospitalized and Recovered Humans
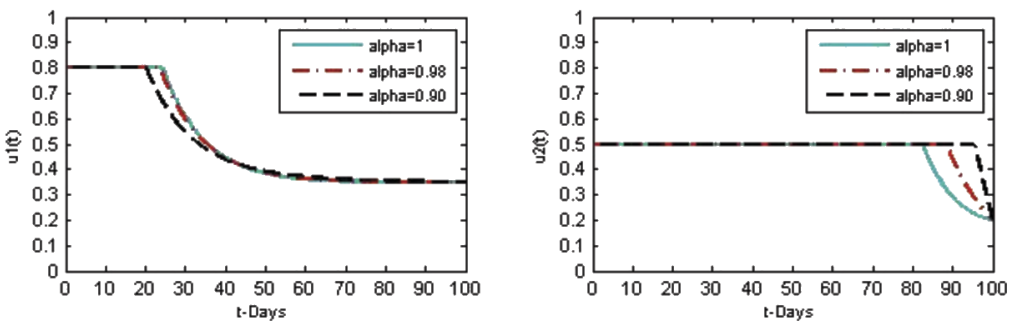

(d) Control Functions

Figure 6 The approximate solutions of the fractional delay optimal control problem by using NSFDM. When $0 \leq u_{1} \leq 0.8,0 \leq u_{2} \leq 0.5$, with $\mathbf{d}=5$ and different $\alpha$, and $\Delta t=0.1$

tiveness of nonstandard finite difference technique for the fractional time-delay optimal control problem are better than the numerical treatments for the time-delay optimal control.

Also, from Table 5 we see that NSFDM helps us to achieve the goal of this research and to obtain the lowest value of the infected and exposed human populations better than SFDM, where the lowest value of these populations occurs at $\alpha=0.90$ and $\mathbf{d}=3,5,7$.

The numerical simulations of the optimal control problem (16) and (17) using two numerical methods, NSFDM and SFDM, are shown in Figs. 1 and 2. Figure 1 presents the comparison between these proposed methods at $\mathbf{d}=10$ and $\alpha=1$, and $\Delta t=1$. It can be observed that the infected human population $I(t)$ is about 280 and 320 for NSFDM and 

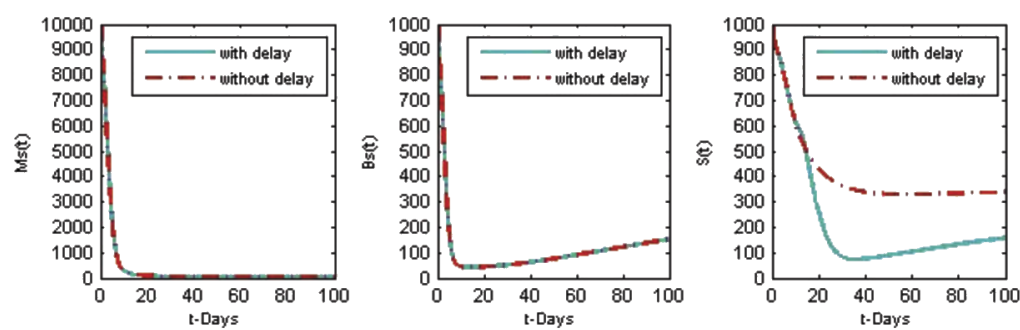

(a) Susceptibles
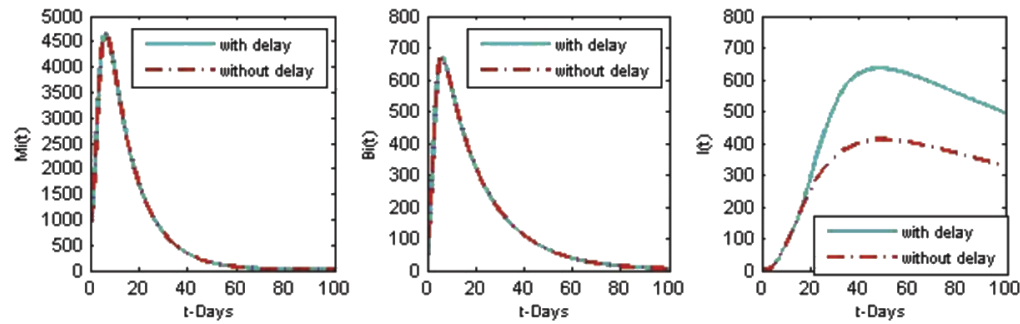

(b) Infected
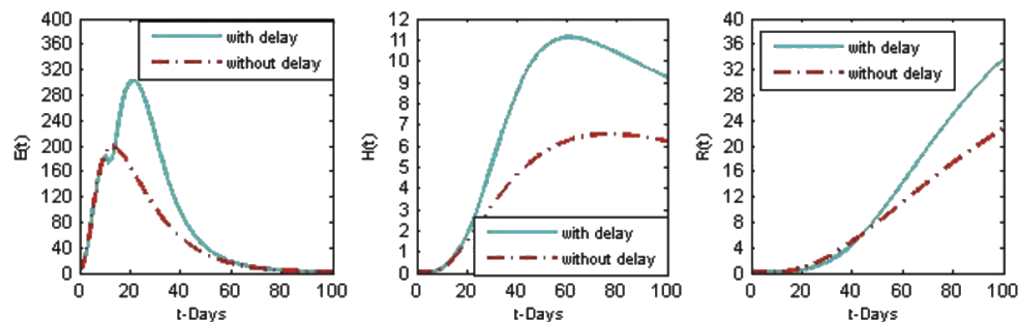

(c) Exposed, Hospitalized and Recovered Humans
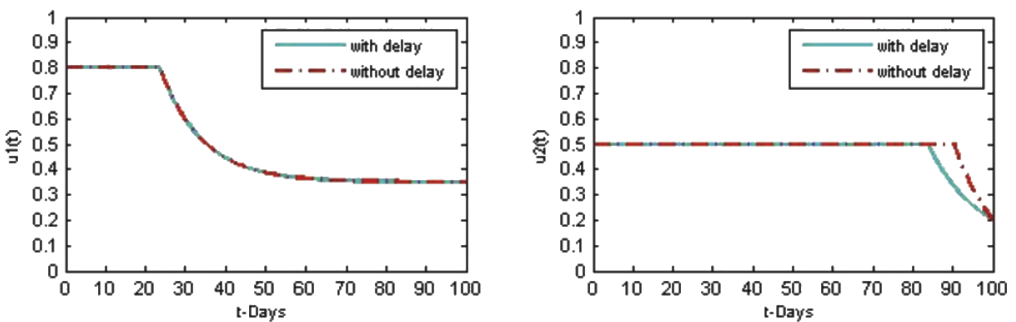

(d) Control Functions

Figure 7 Numerical comparisons of the approximate solutions at $\mathbf{d}=10$ and $\mathbf{d}=0$ by using NSFDM. The approximate solutions of the fractional delay optimal control problem at $\mathbf{d}=10$ and the fraction non-delay optimal control problem at $\mathbf{d}=0$ when $\alpha=0.98$, and $\Delta t=0.1$

SFDM, respectively. It is clear that SFDM is divergent at $\Delta t=5$, but NSFDM is convergent as explained in Figs. 2 and 3, respectively. The approximate solutions of the proposed optimal control problem are described by Figs. 3-9 at different time delay $\mathbf{d}$ and $\alpha$. In Fig. 4, the approximate solutions of the fractional delay WNV model (6) without control (i.e., $u_{1}=u_{2}=0$ ) with $\mathbf{d}=1$ at different $\alpha$, and $\Delta t=1$ by using NSFDM are shown. It can be seen that the infected human population $I(t)$ is about 750, 650, and 400 for $\alpha=1, \alpha=0.98$, and $\alpha=0.90$, respectively. Numerical comparisons between the approximate solutions of the fractional delay optimal control problem (16) and (17) with and without control (i.e., $u_{1}=u_{2}=0$ ) with $\mathbf{d}=1$ at $\alpha=0.90$, and $\Delta t=1$ by using NSFDM, are introduced in Fig. 5. It can be shown that the infected human population $I(t)$ is about 250 and 450 in two cases 

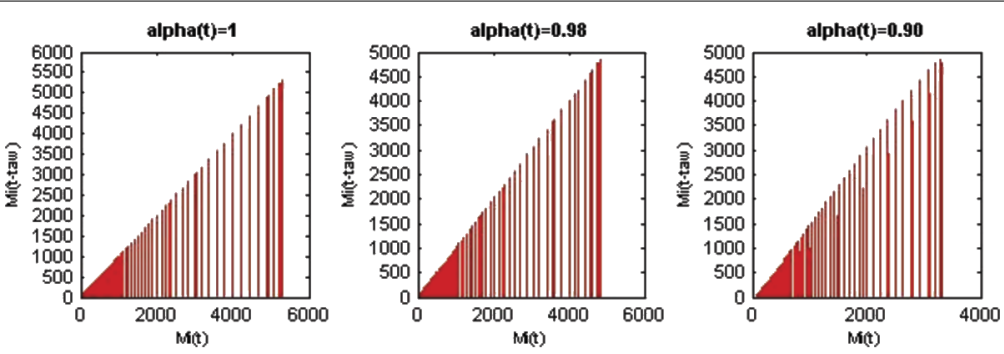

(a) Infected Mosquitoes
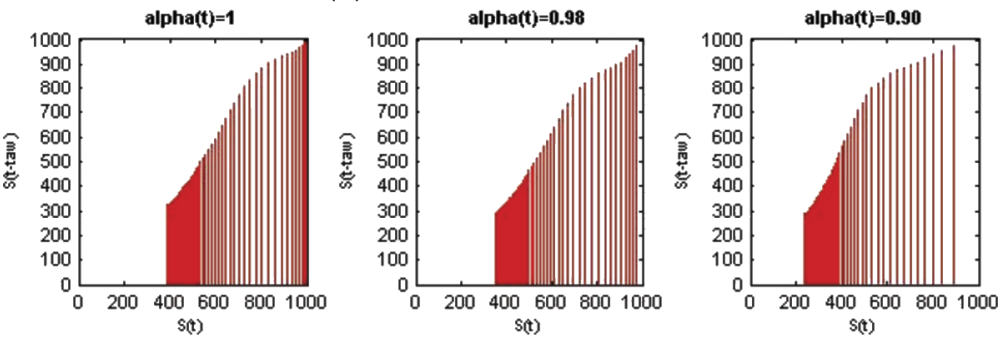

(b) Suscepible Humans
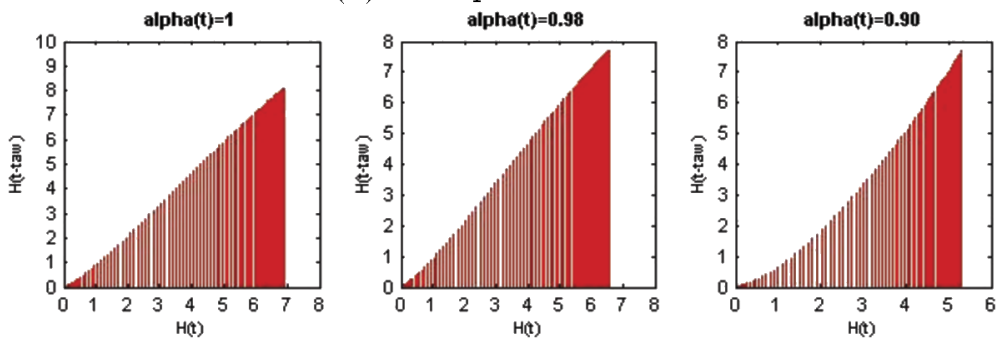

(c) Hospitalized Humans
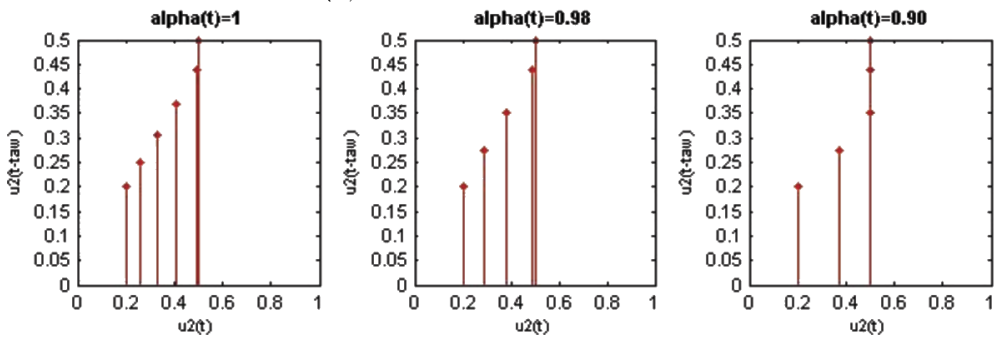

(d) Control Function

Figure 8 The relationship between the delayed variables and the non-delayed variables by using NSFDM. The delayed variables $M_{i}(t-\mathbf{d}), S(t-\mathbf{d}), H(t-\mathbf{d}), u_{2}(t-\mathbf{d})$ and the non-delayed variables $M_{i}(t), S(t), H(t), u_{2}(t)$, respectively, with $\mathbf{d}=5$ and different $\alpha, \Delta t=1$

with and without control, respectively. The behavior of the approximate solutions of the fractional delay optimal control problem (16) and (17) at different time delay $\mathbf{d}$ and different $\alpha$ at $\Delta t=0.1$ is included in Fig. 6 . We notice that the delayed infected human population $I(t-\mathbf{d})$ is about 589.0570, 507.9876, and 278.5177 for $\alpha=1, \alpha=0.98$, and $\alpha=0.90$, respectively, when $\mathbf{d}=5$. Figure 7 shows numerical comparisons between the approximate solutions of the optimal control problem (16) and (17) in two cases, the fractional delay optimal control problem at $\mathbf{d}=5$, and the fraction non-delay optimal control problem at $\mathbf{d}=0$ when $\alpha=0.98$. The infected human population $I(t)$ is about 650 and 400 in two cases with and without delay, respectively. Figure 8 shows the relationship between state 

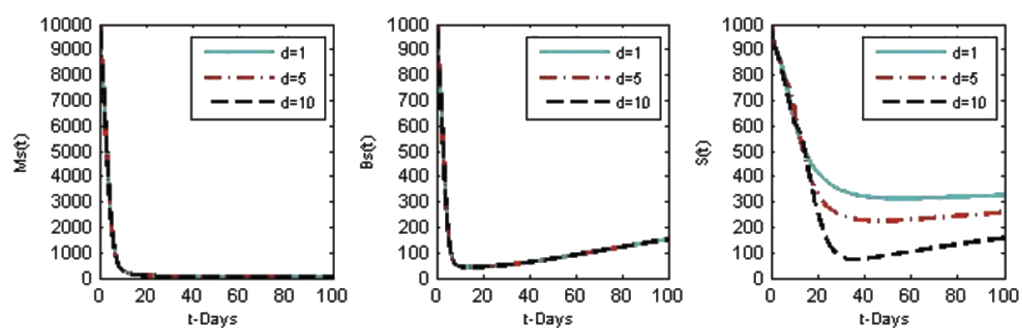

(a) Susceptibles
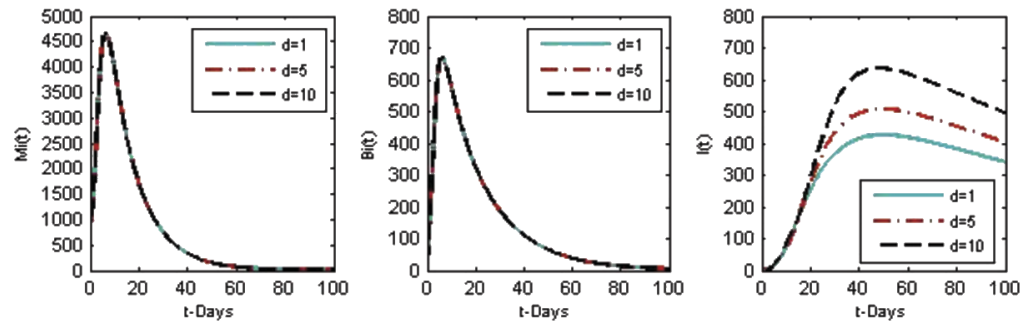

(b) Infected
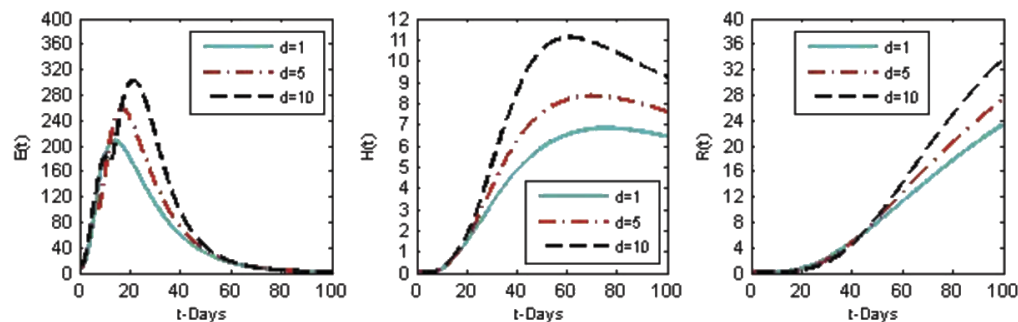

(c) Exposed, Hospitalized and Recovered Humans
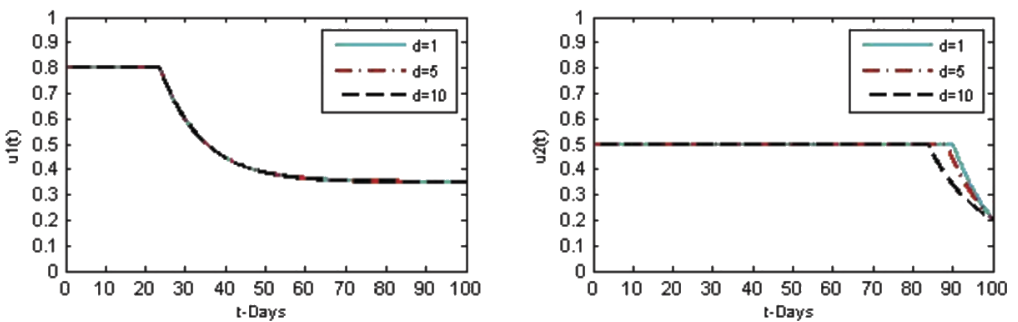

(d) Control Functions

Figure 9 The approximate solutions of the fractional delay optimal control problem by using NSFDM. When $\alpha=0.98$ and different $\mathbf{d}$, and $\Delta t=0.1$

variables $M_{i}(t-\mathbf{d})$ and $M_{i}(t), S(t-\mathbf{d})$ and $S(t), H(t-\mathbf{d})$ and $H(t)$, and the control variable $u_{2}(t-\mathbf{d})$ and $u_{2}(t)$ when $\mathbf{d}=1$ for $\alpha=0.98$. Figure 9 describes the approximate solutions of the fractional delay optimal control problem at the same time delay $\mathbf{d}=1, \mathbf{d}=5$, and $\mathbf{d}=10$ in case of fractional order at $\alpha=0.98$. The infected human population $I(t)$ is about 400,500 , and 650 for $\mathbf{d}=1, \mathbf{d}=5$, and $\mathbf{d}=10$, respectively.

\section{Conclusions}

In this paper, fractional time-delay optimal control in transmission dynamics of WNV model is presented. Time delays in both state and control variables are formulated and studied. NSFDM and SFDM are introduced to study the behavior of the proposed model. According to the results implemented in this paper, it can be observed that the numerical 
Table 4 Comparisons between the value of objective function $J^{*}(\mathfrak{X}, \mathfrak{U})$ with and without control cases and $E\left(T_{f}\right)+I\left(T_{f}\right)$ when $0 \leq u_{1} \leq 0.8,0 \leq u_{2} \leq 0.5$, at different time delay $\mathbf{d}$ and different $\alpha$ with $\Delta t=0.1, T_{f}=100$ by using NSFDM

\begin{tabular}{|c|c|c|c|c|}
\hline $\bar{d}$ & $\alpha$ & $J^{*}(\mathfrak{X}, \mathfrak{U})$ with control & $E\left(T_{f}\right)+I\left(T_{f}\right)$ & $\begin{array}{l}J^{*}(\mathfrak{X}, \mathfrak{U}) \text { without control } \\
\left.\text { (i.e., } u_{1}=u_{2}=0\right)\end{array}$ \\
\hline$\overline{0}$ & $\begin{array}{l}1 \\
0.98 \\
0.90 \\
0.80 \\
0.70\end{array}$ & $\begin{array}{r}43,256 \\
37,592 \\
21,107 \\
9867.9 \\
4376.3\end{array}$ & $\begin{array}{r}389.4656 \\
329.6513 \\
165.8036 \\
66.7680 \\
24.9857\end{array}$ & $\begin{array}{c}68,650 \\
59,318 \\
32,751 \\
15,225 \\
6859.5\end{array}$ \\
\hline 1 & $\begin{array}{l}1 \\
0.98 \\
0.90 \\
0.80\end{array}$ & $\begin{array}{l}44,507 \\
38,732 \\
21,901 \\
10,397\end{array}$ & $\begin{array}{r}403.6675 \\
341.7494 \\
172.1452 \\
69.6851\end{array}$ & $\begin{array}{l}68,771 \\
59,450 \\
32,911 \\
15,401\end{array}$ \\
\hline 3 & $\begin{array}{l}0.70 \\
1 \\
0.98 \\
0.90 \\
0.80 \\
0.70\end{array}$ & $\begin{array}{c}4759.8 \\
47,716 \\
41,612 \\
23,752 \\
11,465 \\
5390.6\end{array}$ & $\begin{array}{r}26.4368 \\
437.1639 \\
370.0171 \\
186.2351 \\
75.5492 \\
28.9362\end{array}$ & $\begin{array}{c}7042.2 \\
69,281 \\
59,951 \\
33,360 \\
15,767 \\
7320.8\end{array}$ \\
\hline 5 & $\begin{array}{l}1 \\
0.98 \\
0.90 \\
0.80 \\
0.70\end{array}$ & $\begin{array}{c}52,063 \\
45,446 \\
26,022 \\
12,616 \\
5981.6\end{array}$ & $\begin{array}{r}478.2057 \\
404.2531 \\
202.3538 \\
81.6707 \\
31.2947\end{array}$ & $\begin{array}{c}70,195 \\
60,795 \\
33,950 \\
16,120 \\
7517.1\end{array}$ \\
\hline 7 & $\begin{array}{l}1 \\
0.98 \\
0.90 \\
0.80 \\
0.70\end{array}$ & $\begin{array}{c}56,867 \\
49,617 \\
28,350 \\
13,721 \\
6521.5\end{array}$ & $\begin{array}{r}522.7261 \\
440.9938 \\
218.8961 \\
87.6141 \\
33.4921\end{array}$ & $\begin{array}{c}71,011 \\
61,304 \\
34,564 \\
16,123 \\
8195.7\end{array}$ \\
\hline
\end{tabular}

Table 5 Comparisons between the value of objective function $J^{*}(\mathfrak{X}, \mathfrak{U})$ using NSFDM and SFDM when $0 \leq u_{1} \leq 0.8,0 \leq u_{2} \leq 0.5$, at different time delay $\mathbf{d}$ and different $\alpha$ with $\Delta t=1, T_{f}=100$

\begin{tabular}{clll}
\hline $\mathbf{d}$ & Method & $\alpha$ & $J^{*}(\mathfrak{X}, \mathfrak{U})$ \\
\hline 3 & NSFDM & 1 & 44,560 \\
& & 0.98 & 40,721 \\
& & 0.90 & 27,976 \\
& SFDM & 1 & 45,647 \\
& & 0.98 & 41,616 \\
& & 0.90 & 28,328 \\
5 & NSFDM & 1 & 46,577 \\
& & 0.98 & 42,650 \\
& & 0.90 & 29,538 \\
& SFDM & 1 & 49,845 \\
& & 0.98 & 45,501 \\
& & 0.90 & 31,115 \\
7 & NSFDM & 1 & 49,176 \\
& & 0.98 & 45,103 \\
& & 0.90 & 31,419 \\
& SFDM & 1 & 54,946 \\
& & 0.98 & 50,146 \\
& & 0.90 & 34,238 \\
10 & NSFDM & 1 & 53,826 \\
& & 0.98 & 49,412 \\
& & 0.90 & 34,499 \\
& SFDM & 1 & 62,325 \\
& & 0.98 & 56,813 \\
& & 0.90 & 38,610 \\
\hline & & &
\end{tabular}


solutions of the proposed model are different when $\mathbf{d}$ and $\alpha$ take different values. From the comparisons included in Tables 4 and 5 , it is found that the values of the objective functional obtained by NSFDM are better than the results obtained by SFDM, it can be applied simply and effectively to solve optimal control of fractional delay.

\section{Acknowledgements}

We thank Seham Mahyoub AL-Mekhlafi for helpful advice during the writing of this work.

\section{Funding}

Not applicable.

\section{Availability of data and materials}

The data sets used and/or analysed during the current study are included in these published articles [26] and [57].

\section{Competing interests}

The authors declare that they have no competing interests.

\section{Authors' contributions}

The authors contributed equally to this paper. All authors read and approved the final manuscript.

\section{Author details}

'Department of Mathematics, Faculty of Science, Cairo University, Giza, Egypt. ${ }^{2}$ Department of Mathematics, Faculty of Science, Helwan University, Cairo, Egypt.

\section{Publisher's Note}

Springer Nature remains neutral with regard to jurisdictional claims in published maps and institutional affiliations.

Received: 25 September 2018 Accepted: 20 May 2019 Published online: 29 May 2019

\section{References}

1. Chen, B.S., Wang, S.S., Lu, H.C.: Stabilization of time-delay systems containing saturating actuators. Int. J. Control 47(3), 867-881 (1988)

2. Chyung, D.H., Lee, E.B.: Linear optimal systems with time-delay. SIAM J. Control 4(3), 548-575 (1966)

3. Lee, T.N., Radovic, U.L.: General decentralized stabilization of large-scale linear continuous and discrete time-delay systems. Int. J. Control 46(3), 2127-2140 (1987)

4. Yang, J.N., Soong, T.T.: Recent advances in active control of civil engineering structures. Probab. Eng. Mech. 3(4), 179-188 (1988)

5. Cai, G.P., Huang, J.Z., Yang, S.X.: An optimal control method for linear systems with time delay. Comput. Struct. 81, 1539-1546 (2003)

6. Wong, K.H., Clements, D.J., Teo, K.L.: Optimal control computation for nonlinear time-lag systems. J. Optim. Theory Appl. 47(1), 91-107 (1985)

7. Teo, K.L., Wong, K.H., Clements, D.J.: Optimal control computation for linear time-lag systems with linear terminal constraints. J. Optim. Theory Appl. 44(3), 509-526 (1984)

8. Dadebo, S., Luus, R.: Optimal control of time-delay systems by dynamic programming. Optim. Control Appl. Methods 13, 29-41 (1992)

9. Luus, R., Zhang, X., Hartig, F., Keil, F.J.: Use of piecewise linear continuous optimal control for time-delay systems. Ind. Eng. Chem. Res. 34, 4136-4139 (1995)

10. Oğuztöreli, M.N.: Time-Lag Control Systems. Academic Press, New York (1966)

11. Kharatishvili, G.L.: A maximum principle in the theory of optimal time-delay processes. Dokl. Akad. Nauk SSSR 136, 39-42 (1961)

12. Kharatishvili, G.L.: A Maximum Principle in External Problems with Delays. Mathematical Theory on Control. Academic Press, New York (1967)

13. Halanay, A.: Optimal controls for systems with time lag. SIAM J. Control 6, 215-234 (1968)

14. Soliman, M.A., Ray, W.H.: On the optimal control of systems having pure time delays and singular arcs. Int. J. Control 16(5), 963-976 (1972)

15. Guinn, T:: Reduction of delayed optimal control problems to nondelayed problems. J. Optim. Theory Appl. 18, 371-377 (1976)

16. Banks, H.T.: Necessary conditions for control problems with variable time lags. SIAM J. Control 8(1), 9-47 (1968)

17. Colonius, F., Hinrichsen, D.: Optimal control of functional differential systems. SIAM J. Control Optim. 16(6), 861-879 (1978)

18. Sweilam, N.H., Al-Mekhlafi, S.M.: Optimal control for a time delay multi-strain tuberculosis fractional model: a numerical approach. IMA J. Math. Control Inf. 36, 317-340 (2019) https://doi.org/10.1093/imamci/dnx046

19. Jajarmi, A., Pariz, N., Effati, S., Vahidian Kamyad, A.: Solving infinite horizon nonlinear optimal control problems using an extended modal series method. J. Zhejiang Univ.-Sci. C, Comput. Electron. 12(8), 667-677 (2011)

20. Jajarmi, A., Pariz, N., Effati, S., Vahidian Kamyad, A.: Infinite horizon optimal control for nonlinear interconnected large-scale dynamical systems with an application to optimal attitude control. Asian J. Control 14(5), 1239-1250 (2012) 
21. Jajarmi, A., Hajipour, M., Mohammadzadeh, E., Baleanu, D.: An efficient recursive shooting method for the optimal control of time-varying systems with state time-delay. Appl. Math. Model. 40(4), 2756-2769 (2016). https://doi.org/10.1016/j.apm.2015.09.072

22. Sweilam, N.H., Al-Mekhlafi, S.M.: Comparative study for multi-strain tuberculosis (TB) model of fractional order. Appl. Math. Inf. Sci. 10(4), 1403-1413 (2016)

23. Sweilam, N.H., Al-Mekhlafi, S.M.: Numerical study for multi-strain tuberculosis (TB) model of variable-order fractional derivatives. J. Adv. Res. 7(2), 271-283 (2016)

24. Sweilam, N.H., Al-Mekhlafi, S.M.: On the optimal control for fractional multi-strain TB model. Optim. Control Appl. Methods 37(6), 1355-1374 (2016)

25. Jajarmi, A., Hajipour, M., Mohammadzadeh, E., Baleanu, D.: A new approach for the nonlinear fractional optimal control problems with external persistent disturbances. J. Franklin Inst. 335(9), 3938-3967 (2018). https://doi.org/10.1016/j.jfranklin.2018.03.012

26. Sweilam, N.H., Saad, O.M., Mohamed, D.G.: Comparative studies for the fractional optimal control in transmission dynamics of West Nile virus. Int. J. Biomath. 10(7), 1-31 (2017). https://doi.org/10.1142/S1793524517500954

27. Nagy, A.M., Sweilam, N.H.: An efficient method for solving fractional Hodgkin-Huxley model. Phys. Lett. A 378(30-31), 1980-1984 (2014)

28. Sweilam, N.H., Khader, M.M., Mahdy, A.M.S.: Numerical studies for fractional-order logistic differential equation with two different delays. J. Appl. Math. 2012, Article ID 764894 (2012)

29. Sweilam, N.H., Khader, M.M., Adel, M.: On the stability analysis of weighted average finite difference methods for fractional wave equation. Fract. Differ. Calc. 2(1), 17-29 (2012)

30. Sweilam, N.H., Khader, M.M., Nagy, A.M.: Numerical solution of two-sided space-fractional wave equation using finite difference method. J. Comput. Appl. Math. 235(8), 2832-2841 (2011)

31. Jajarmi, A., Baleanu, D.: Suboptimal control of fractional-order dynamic systems with delay argument. J. Vib. Control 24(12), 2430-2446 (2018)

32. Sweilam, N.H., Al-Mekhlafi, S.M., Assiri, T.A.: Numerical study for time delay multistrain tuberculosis model of fractional order. Complexity 2017, Article ID 1047384 (2017)

33. Rihan, F.A., Hashish, A.H., Al-Maskari, F., Hussein, M.S., Ahmed, E., et al.: Dynamics of tumor-immune system with fractional-order. J. Tumor Res. 2, Article 109 (2016)

34. Rihan, F.A., Lakshmanan, S., Hashish, A.H., Rakkiyappan, R., Ahmed, E.: Fractional-order delayed predator-prey systems with Holling type-II functional response. Int. J. Nonlinear Dyn. Chaos Eng. Syst. 80(1-2), 777-789 (2015)

35. Alligood, K.T., Sauer, T.D., Yorke, J.A.: Chaos: An Introduction to Dynamical Systems. Springer, Berlin (1997)

36. Daftardar-Gejji, V., Sukale, Y., Bhalekar, S.: Solving fractional delay differential equations: a new approach. Fract. Calc. Appl. Anal. 18(2), 400-418 (2015)

37. Wang, Z., Huang, X., Zhou, J.: A numerical method for delayed fractional-order differential equations: based on G-L definition. Appl. Math. Inf. Sci. 7(2), 525-529 (2013)

38. Rihan, F.A.: Computational methods for delay parabolic and time-fractional partial differential equations. Numer. Methods Partial Differ. Equ. 26(6), 1556-1571 (2010)

39. Mickens, R.: Nonstandard Finite Difference Models of Differential Equations. World Scientific, Singapore (1994)

40. González-Parra, G., Arenas, A.J., Chen-Charpentier, B.M.: Combination of nonstandard schemes and Richardson's extrapolation to improve the numerical solution of population models. Math. Comput. Model. 54, 1030-1036 (2010)

41. Mickens, R.E.: Calculation of demoninator functions for nonstandard finite difference scheme for differential equations satisfying a positivity condition. Numer. Methods Partial Differ. Equ. 23, 672-691 (2007)

42. Abraham Arenas, J., Gilberto González-Parra, G., Benito Chen-Charpentier, M.: Construction of nonstandard finite difference schemes for the SI and SIR epidemic models of fractional order. Math. Comput. Simul. 121, 48-63 (2016)

43. Bowman, C., Gumel, A.B., Van den Driessche, P., Wu, J., Zhu, H.: A mathematical model for assessing control strategies against West Nile virus. Bull. Math. Biol. 67, 1107-1133 (2005)

44. Fan, G., Liu, J., Van den Driessche, P., Wu, J., Zhu, H.: The impact of maturation delay if mosquitoes on the transmission of West Nile virus. Math. Biosci. 228, 119-126 (2010)

45. Smithburn, K.C., Hughes, T.P., Burke, A.W., Paul, J.H.: A neurotropic virus isolated from the blood of native of Uganda. Am. J. Trop. Med. Hyg. 20, 471-492 (1940)

46. Campbell, G.L., Marfin, A.A., Lanciotti, R.S., Gubler, D.J.: West Nile virus: reviews. Lancet Infect. Dis. 2, 519-529 (2002)

47. (2002) Center for Disease Control and Prevention (CDC): West Nile virus: virus history and distribution. http://www.cdc.gov/ncidod/dvbid/westnile/background.html

48. Chowers, M.Y., Lang, R., Nassar, F., et al.: Clinical characteristics of the West Nile fever outbreak, Israel, 2000. Emerg. Infect. Dis. 7, 686-691 (2001)

49. Nash, D., Mostashari, F., Fine, A., et al.: The outbreak of West Nile virus infection in the New York City area in 1999. N. Engl. J. Med. 344, 1807-1814 (2001)

50. Peter, L.R., Marfin, A.A.: West Nile virus: a primer for the clinician. Ann. Intern. Med. 137, 173-179 (2002)

51. Thomas, M., Urena, B.: A model describing the evolution of West Nile-like encephalitis in New York City. Math. Comput. Model. 34, 771-781 (2001)

52. Wonhman, M., de-Camino-Beck, T., Lewis, M.: An epidemiological model for West Nile virus: invasion analysis and control applications. Proc. R. Soc. Lond. B, Biol. Sci. 271, 501-507 (2004)

53. Cruz-Pacheco, G., Esteva, L., Montano-Hirose, J., Vargas, D.: Modelling the dynamics of West Nile virus. Bull. Math. Biol. 67, 1157-1172 (2005)

54. Lewis, M., Renclawowicz, J., van den Driessche, P.: Traveling waves and spread rates for a West Nile virus model. Bull. Math. Biol. 66, 3-23 (2006)

55. Wonhman, M., Lewis, M., Rencawowicz, J., Van den Driessche, P.: Transmission assumptions generate conflicting predictions in hostvector disease models: a case study in West Nile virus. Ecol. Lett. 9, 706-725 (2006)

56. Lewis, M., Renclawowicz, J., van den Driessche, P., Wonhman, M.: A comparison of continuous and discrete-time West Nile virus models. Bull. Math. Biol. 68, 491-509 (2006)

57. Kbenesh Blayneh, W., Gumel, A.B., Lenhart, S., Clayton, T.: Backward bifurcation and optimal control in transmission dynamics of West Nile virus. Bull. Math. Biol. 72, 1006-1028 (2010) 
58. Podlubnny, I.: Fractional Differential Equations. Academic Press, New York (1999)

59. Anguelov, R., Lubuma, J.M.S.: Nonstandard finite difference method by nonlocal approximation. Math. Comput. Simul. 61(36), 465-475 (2003)

60. Mickens, R.: Application of Nonstandard Finite Difference Schemes. World Scientific, Singapore (2000)

61. Carvalho, A., Pinto, C.M.A.: A delay fractional order model for the co-infection of malaria and HIV/AIDS. Int. J. Dyn. Control 10, 168-186 (2017)

62. El-Saka, H.A.A.: The fractional-order SIR and SIRS epidemic models with variable population. Math. Sci. Lett. 2(3), 195-200 (2013)

63. Matignon, D.: Stability results for fractional differential equations with applications to control processing. In: Computational Engineering in Systems and Application. Multiconference, IMACS, IEEE-SMC, Lille, France 2, pp. 963-968. (1996)

64. Göllmann, L., Maurer, H.: Theory and applications of optimal control problems with multiple time-delays. Special issue on computational methods for optimization and control. J. Ind. Manag. Optim. 10, 413-441 (2014)

\section{Submit your manuscript to a SpringerOpen ${ }^{\circ}$} journal and benefit from:

- Convenient online submission

Rigorous peer review

- Open access: articles freely available online

- High visibility within the field

- Retaining the copyright to your article

Submit your next manuscript at $\boldsymbol{\Delta}$ springeropen.com 